\title{
1 Smooth muscle-specific MMP17 (MT4-MMP) defines the intestinal ECM niche
}

2

3 Mara Martín-Alonso ${ }^{1 *}$, Håvard T. Lindholm ${ }^{1, \#}$, Sharif lqbal ${ }^{2,3, \#}$, Pia Vornewald ${ }^{1 \#}$, Sigrid Hoel ${ }^{1}$,

4 Mirjam J. Damen ${ }^{4}$, A.F.Maarten Altelaar ${ }^{4}$, Pekka Katajisto ${ }^{2,3,5}$, Alicia G. Arroyo ${ }^{6,7}$, Menno J.

5 Oudhoff $^{1 *}$

6

$7 \quad{ }^{1}$ CEMIR - Centre of Molecular Inflammation Research, Department of Clinical and Molecular

8 Medicine, NTNU - Norwegian University of Science and Technology, 7491 Trondheim,

9 Norway

$10{ }^{2}$ Institute of Biotechnology, HiLIFE, University of Helsinki, Finland

$11{ }^{3}$ Molecular and Integrative Bioscience Research Programme, Faculty of Biological and

12 Environmental Sciences, University of Helsinki, Helsinki, Finland

$13{ }^{4}$ Biomolecular Mass Spectrometry and Proteomics, Utrecht University, Utrecht, The

14 Netherlands

$15{ }^{5}$ Department of Biosciences and Nutrition, Karolinska Institutet, Stockholm, Sweden

\#These authors contributed equally to this work

*corresponding authors, e-mail: mara.m.alonso@ntnu.no, menno.oudhoff@ntnu.no 


\section{SUMMARY ( 150 words)}

Smooth muscle is an essential component of the intestine, both to maintain its structure and produce peristaltic and segmentation movements. However, very little is known about other putative roles that smooth muscle may have. Here, we show that smooth muscle is the dominant supplier of BMP antagonists, which are niche factors that are essential for intestinal stem cell maintenance. Furthermore, muscle-derived factors can render epithelium reparative and fetal-like, which includes heightened YAP activity. Mechanistically, we find that the matrix metalloproteinase MMP17, which is exclusively expressed by smooth muscle, is required for intestinal epithelial repair after inflammation- or irradiation-induced injury. Furthermore, we provide evidence that MMP17 affects intestinal epithelial reprogramming indirectly by cleaving the matricellular protein PERIOSTIN, which itself is able to activate YAP. Together, we identify an important signaling axis that firmly establishes a role for smooth muscle as a modulator of intestinal epithelial regeneration and the intestinal stem cell niche.

\section{INTRODUCTION}

The intestinal epithelium consists of a single layer of cells that is important for the uptake of nutrients as well as for providing a barrier to protect from pathogens. During homeostasis, intestinal epithelial cells are all derived from LGR5+ intestinal stem cells (ISCs) that reside at the bottom of crypts (Barker et al. 2007). Upon injury, however, or after depletion of LGR5+ cells, LGR5- cells can rapidly regain LGR5 expression and thus dedifferentiate to repopulate the crypt bottoms (Tian et al. 2011; Murata et al. 2020). In addition, within this dedifferentiation process, an epithelial reparative state exists, which is fetal-like, and depends on reprogramming by YAP (Yui et al. 2018; Gregorieff et al. 2015), and is further characterized by markers such as SCA-1 and HOPX (Wang et al. 2019; Nusse et al. 2018; Yui et al. 2018)

Adult intestinal epithelial (stem cell) maintenance relies on a variety of niche factors such as WNTs, R-spondins (RSPOs), Bone morphogenic proteins (BMPs), and prostaglandins, all of which are expressed by mesenchymal cell subtypes (McCarthy et al. 2020; Miyoshi et al. 2017; Greicius et al. 2018b; Shoshkes-Carmel et al. 2018a; Roulis et al. 2020; Degirmenci et al. 2018). These mesenchymal cells reside in the mucosa near the epithelium. However, intestinal epithelial homeostasis does not solely rely on soluble niche factors. The mechanical or extracellular matrix (ECM) niche is an additional defining factor, for example, by modulating the mechanosensory HIPPO/YAP pathway (Yui et al. 2018; Gjorevski et al. 2016). In addition, growth factors can interact with, or be embedded within the ECM to 
modulate their activity. Smooth muscle cells are one of the most prevalent non-epithelial cell types throughout the intestine, yet, their role in providing niche factors or affecting the ECM niche is largely unknown. Nevertheless, smooth-muscle specific deletion of tumor suppressor genes can result in defective epithelial growth (Katajisto et al. 2008). Furthermore, it was shown that cells that originated from the smooth-muscle can migrate into the mucosa to aid after injury (Chivukula et al. 2014). Nevertheless, it is still largely undefined what role adult smooth muscle has in ISC maintenance or during repair after injury.

Matrix metalloproteinases (MMPs) are fundamental ECM regulators, both by modifying ECM components directly and by cleaving growth factors to control their ability to bind the ECM and to the cell (Page-McCaw, Ewald, and Werb 2007a; Kessenbrock, Wang, and Werb 2015; Martín-Alonso et al. 2015). MMPs can play various roles in the injured intestine and many MMPs are upregulated in inflammatory bowel disease, likely by the increase in immune cell populations such as neutrophils with high proteolytic activity or in endothelial cells (O'Sullivan, Gilmer, and Medina 2015; Esteban et al. 2020) . Thus, inhibition of MMP activity may be an attractive therapeutic target for treating inflammatory bowel disease (Jakubowska et al. 2016; Esteban et al. 2020). Although the role of certain MMPs such as MMP2, MMP7, MMP9, and MT1-MMP are relatively well-studied, the role of other MMPs in intestinal biology is still largely unknown. Here, we show that MMP17, a membrane-bound MMP which is specifically expressed in smooth muscle, is important to maintain optimal ISC stemness during homeostasis, and preserve the regenerative capacity of intestinal epithelium. 


\section{RESULTS}

\section{Intestinal smooth muscle is a rich source of BMP antagonists}

Based on recent indications that fetal intestinal muscle is a provider of niche factors (Czerwinski et al. 2020), we first separated smooth muscle tissue and epithelial crypts from adult mouse colon and performed RNA-seq (Fig.1a, 1b, S1a). We found little evidence that intestinal smooth muscle expressed niche factors such as WNTs and RSPOs, or growth factors such as epidermal growth factor (EGF), however, we did find that smooth muscle expresses high levels of factors associated with BMP signaling including Grem1, Grem2, and Chrdnl1 (Fig. 1b, S1a). Fluorescent in situ hybridization (FISH) confirmed high levels of these factors in a muscle-specific manner, in particular, we found enrichment of these factors in the muscularis mucosae that resides in close proximity to the bottom of epithelial crypts (Fig. 1c).

\section{Intestinal smooth muscle provides niche factors that render organoid growth independent of NOGGIN}

Intestinal organoids are self-organizing in vitro epithelial structures that can be used to model in vivo processes (Sato et al. 2009; Serra et al. 2019). To test for a functional role of smooth muscle in providing niche factors, we exposed intestinal organoids to supernatant from muscle explants (muscle-SN) that completely lacked the mucosa (Fig. S1b). We found that organoids exposed to muscle-SN grew 2-3 times larger than in standard conditions and had a predominantly spheroid morphology (Figure 1d-g). Intestinal organoids rely on the supplementation of 3 factors epithelium itself does not express sufficiently; EGF, NOGGIN, and RSPO1 (ENR). Thus, we next tested whether muscle-SN could replace these factors. We found that muscle-SN was unable to replace RSPO1, as organoids failed to grow in RSPO1-deficient medium irrespective of muscle-SN supplementation (Figure 1d, 1e). In contrast, we found that muscle-SN was able to substitute for the BMP antagonist NOGGIN (Figure 1d, 1e). This is in support of our RNA-seq data in which we observed low Rspo1/2/3 levels, but ample expression of BMP antagonists such as Grem1 in smooth muscle tissue (Fig. 1b, 1c, S1a). ENR medium supports self-renewal and differentiation of organoids that acquire a typical structure including proliferative budding crypts and non-proliferative villus regions (Fig. 1f, 1g). In contrast, muscle-SN exposed organoids lacked crypts and consisted of mainly proliferative cells that were equally distributed along the spheroid (Fig. 1f, 1g). 

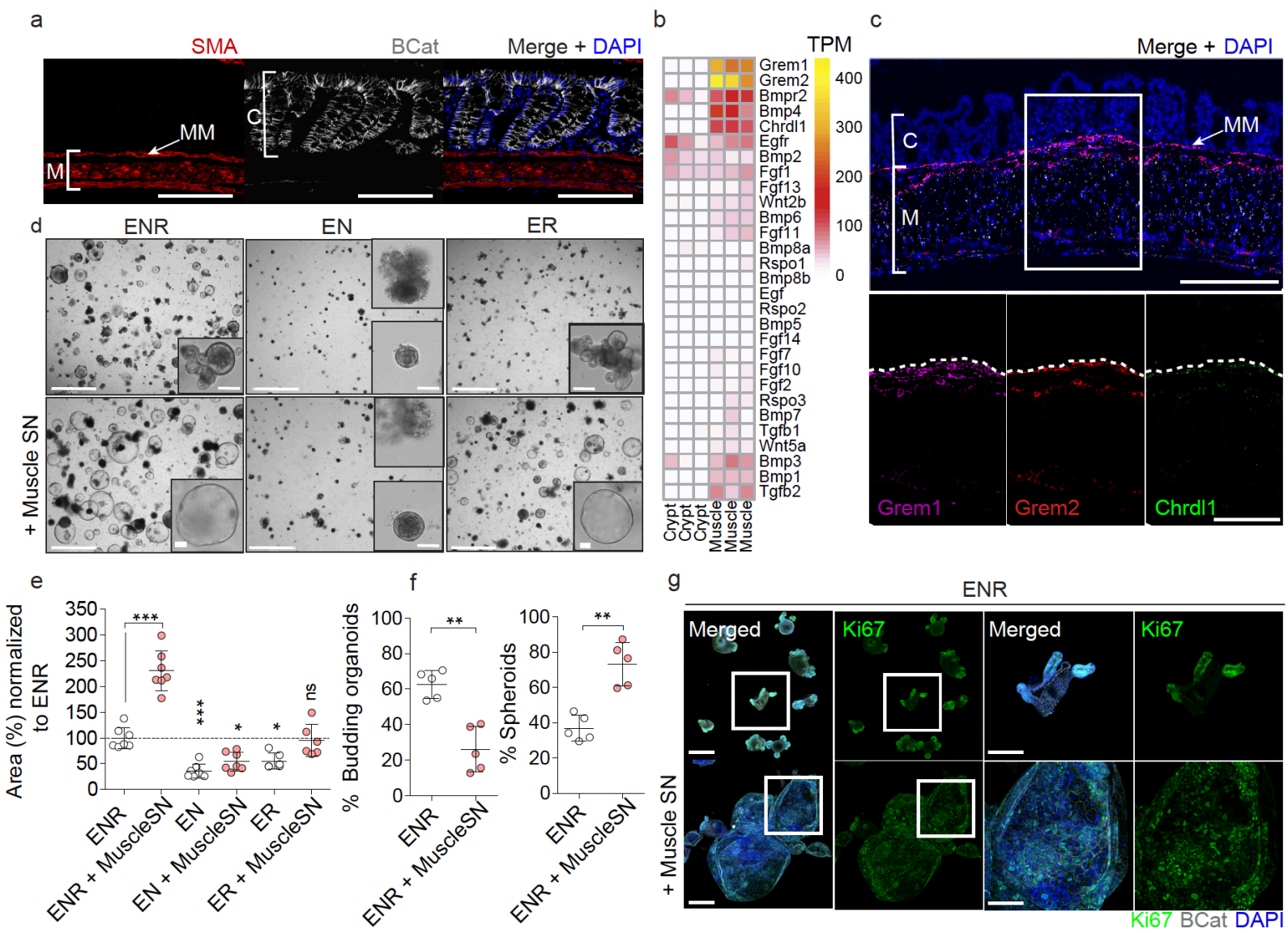

Figure 1. Intestinal Smooth muscle expresses BMP antagonists that control epithelial behavior. a Representative confocal maximum intensity projection of a transverse colon cut showing specific staining for muscle (M), SMA (Red), and crypts (C), $\beta$ Catenin ( $\beta$ cat, Grey). Clear distinction among muscle layers can be observed, from muscularis mucosae (MM) close to the crypts to circular and longitudinal smooth muscle layers. Scale bar $100 \mu \mathrm{m}$. b. Heatmap depicting gene expression levels in TPM (Transcripts per million) in crypts vs muscle intestinal samples. $n=3$ biological replicates. c. Representative confocal maximum intensity projections of fluorescence-coupled RNAscope showing BMP antagonists Grem1 in magenta, Grem2 in red, and Chrdl1 in green, expressed in muscular cells. Scale bar $100 \mu \mathrm{m} ; 50 \mu \mathrm{m}$ in magnified image. $\mathrm{n}=2$ mice. d. Representative bright field pictures showing organoids morphological changes when exposed to muscle-derived factors (Day4). Control media ENR (EGF, NOGGIN, RSPO1), EN (no RSPO1) or ER (no NOGGIN) were used to assess organoids reliance on external growth factors. Scale $1250 \mu \mathrm{m} ; 100 \mu \mathrm{m}$ in magnified image. e. Graph shows organoids area in response to different media. Depicted average values normalized to ENR condition. $n=5$ to 7 independently performed experiments with 2-3 wells/experiment. f. Graphs represents the percentage of budding organoids or spheroids in response to ENR or ENR + muscle supernatant (Muscle SN). $n=5$ independently performed experiments with 2-3 wells/experiment. g. Representative maximun projection confocal images showing proliferative active cells (Ki67) in green and cell shape (ßcat, grey) staining. Scale $200 \mu \mathrm{m}, 100 \mu \mathrm{m}$ in magnified image. $n=3$ independent experiments. Numerical data are means $\pm S D$. Data were analyzed by one-way ANOVA (e) or by Mann Whitney-test (f). 
136 We find that intestinal smooth muscle expresses and secretes soluble niche factors that can 137 mediate epithelial organoid growth (Fig. 1). Next, we wished to determine whether smooth 138 muscle may also affect the ECM. The ECM acts on ISCs by providing a 'mechanical' niche 139 as well as by being a reservoir of ECM-bound niche factors (Meran, Baulies, and Li 2017; 140 Gattazzo, Urciuolo, and Bonaldo 2014; Kessenbrock, Wang, and Werb 2015). MMPs are 141 known as important modulators of the ECM (Page-McCaw, Ewald, and Werb 2007b;

142 Rodríguez, Morrison, and Overall 2010). By directly comparing epithelial crypt and smooth 143 muscle we found several MMPs to be specifically expressed in smooth muscle tissue (Fig.

144 2a), including Mmp17 (also known as MT4-MMP), a previously identified regulator of muscle 145 growth factors and its surrounding ECM (Martín-Alonso et al. 2015). Therefore, we analyzed 146 Mmp17 expression in intestinal tissue by using the $\mathrm{KO} / \mathrm{KI}$ mouse strain Mmp17 ${ }^{\mathrm{LacZ} / \mathrm{LacZ}}$ 147 (referred to as KO mice from hereon) (Rikimaru et al. 2007). We used LacZ staining (Fig.

148 S2a) or a specific anti $\beta$-gal antibody to detect $M m p 17$ promoter activity in the intestine from $149 \mathrm{Mmp}_{17^{\mathrm{LacZ} /+}}$ mice. As shown in Figure $2 \mathrm{~b}, \mathrm{MMP} 7^{+}$cells are located only in the smooth 150 muscle, and we identified that relatively more cells were MMP $17^{+}$in the muscularis mucosae 151 compared to the circular and longitudinal layers of the muscle (Fig. 2b, 2c). This is 152 reminiscent of the expression of BMP antagonists (Fig. 1c), and indeed, using FISH, we find 153 that Mmp17 has an overlapping expression pattern with these BMP antagonists including 154 enrichment in the muscularis mucosae (Fig 2d).
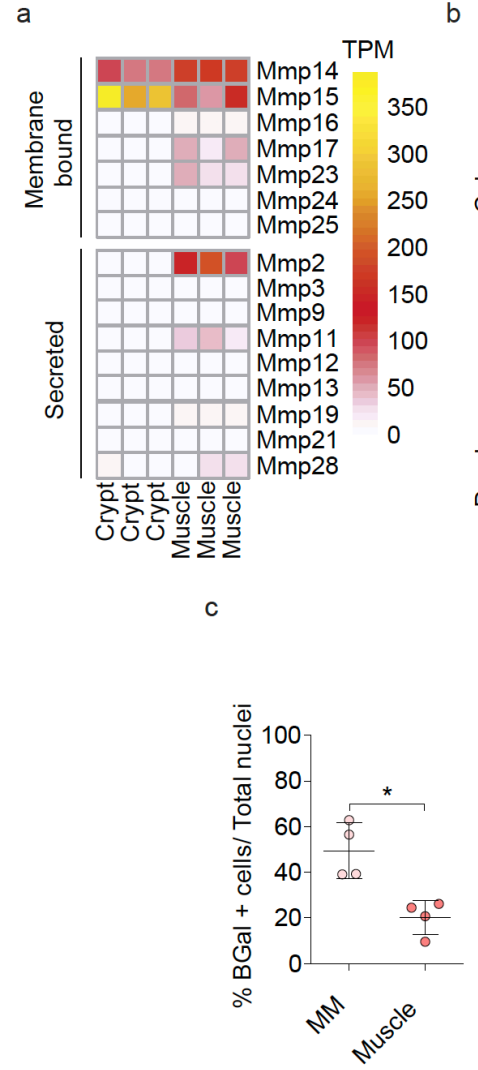

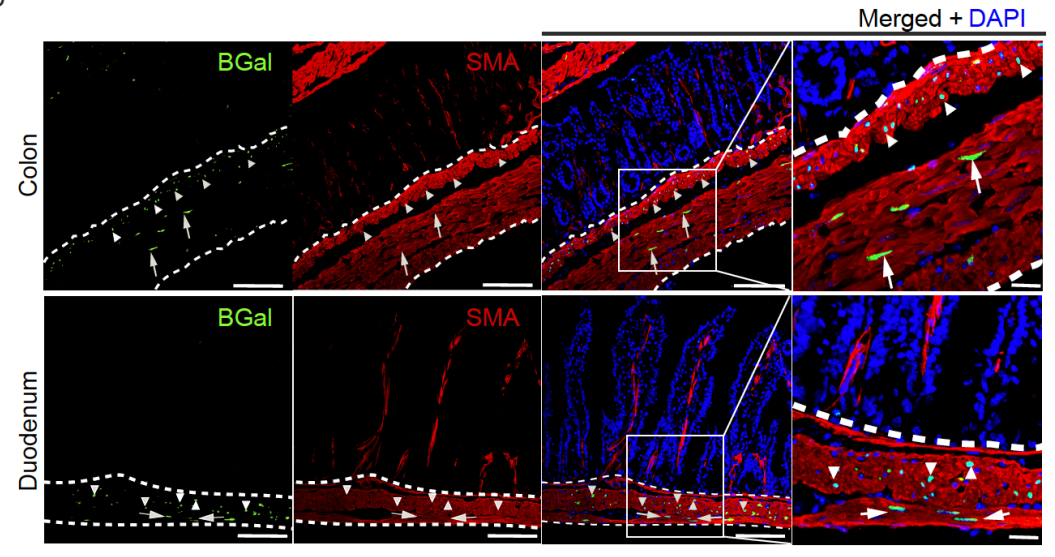

d

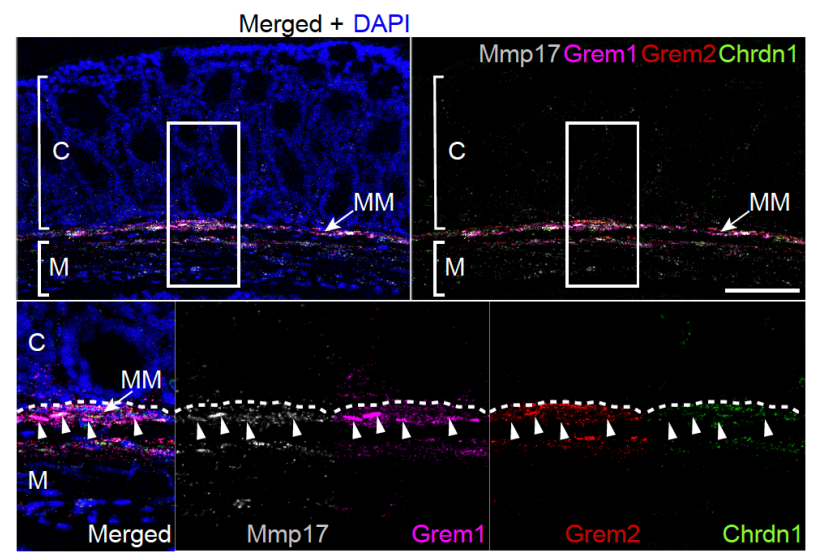


Figure 2. Muscle-specific matrix metalloproteinase Mmp17 is enriched in the muscularis mucosae together with BMP antagonists expressing cells. a. Heatmap shows TPM levels of Matrix Metalloproteinases (MMPs) in crypts vs muscle. $n=3$ biological replicates. b. Representative confocal microscopy images showing active Mmp17 promoter ( $\beta$ Gal staining, green) in positive SMA (red) muscle (white dashed line) stained in transverse intestinal sections of $M m p 17+/-$ mice. Scale bar $100 \mu \mathrm{m} ; 20 \mu \mathrm{m}$ in magnified image. $\mathrm{n}=3$ animals. c. Graph shows quantification of the \% number of $\beta$ Gal positive cells in muscularis mucosae (MM) vs circular and longitudinal muscle (Muscle) in colon samples. $n=3$ biological replicates. 4 images quantified. $d$. Representative confocal maximal projections of fluorescence-coupled RNAscope showing Mmp17 (grey), and BMP antagonists (Grem1, magenta, Grem2, red and Chrdl1, green) co-expression in muscular cells (arrowheads). C, crypts, M, Muscle and MM, muscularis mucosae. Scale bar $100 \mu \mathrm{m} ; 50 \mu \mathrm{m}$ in magnified image. $\mathrm{n}=2$ independent experiments with 1-2 samples/genotype. Numerical data in c are means \pm SD and was analyzed by Mann-Whitney test.

\section{Muscle-specific MMP17 controls BMP signaling in crypts}

Since MMP17 is enriched in the muscularis mucosa where its expression correlates with BMP antagonists, we asked about the possible impact of MMP17 loss in crypts. To unbiasedly gain mechanistic insight, we performed RNA-seq comparing WT and KO colonic smooth muscle and crypts. Mmp17 is expressed in smooth muscle but not in the epithelium (Fig. 2), and we were surprised to find that only 42 genes were dysregulated in the KO muscle whereas 191 genes were dysregulated in KO crypts compared to their WT counterparts (Fig. 3a). In support, principal component analysis (PCA) was unable to distinguish WT from KO muscle, whereas KO crypts had a different distribution compared to WT crypts (Fig. 3b). Furthermore, Mmp17 absence in intestinal smooth muscle did not result in any structural alteration at the smooth muscle level (Figure S3a). Upon closer examination, using the online gene enrichment tool Enrichr (E. Y. Chen et al. 2013; Kuleshov et al. 2016), we found SMAD4 as the top TF associated with upregulated genes in KO crypts (Fig. S3b). To test whether increased SMAD4 target genes in KO crypts was a direct result of SMAD4 protein levels, we performed immunostaining and western blot for SMAD4. Corroborating our unbiased transcriptome analysis, we found heightened nuclear localization of SMAD4 in the bottom of KO crypts compared to WT crypts, and increased levels of total SMAD4 in KO mucosa (Fig. 3d, 3e). The nuclear translocation or overall accumulation of SMAD4 is the result of cellular activation by TGF $\beta$ family members, including BMPs that specifically induce SMAD1/5/9 phosphorylation (Massagué 2012). Indeed, we found pSMAD1/5/9 to be particularly enriched in the bottom of KO crypts compared to WT crypts (Fig. 3f), which suggests altered BMP signalling in KO intestinal epithelium. 
a

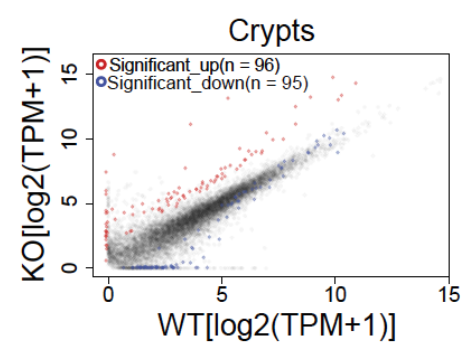

C Enriched Terms Crypts

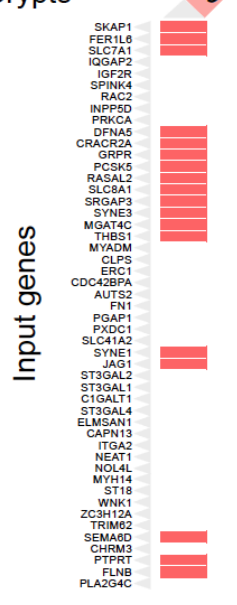

d

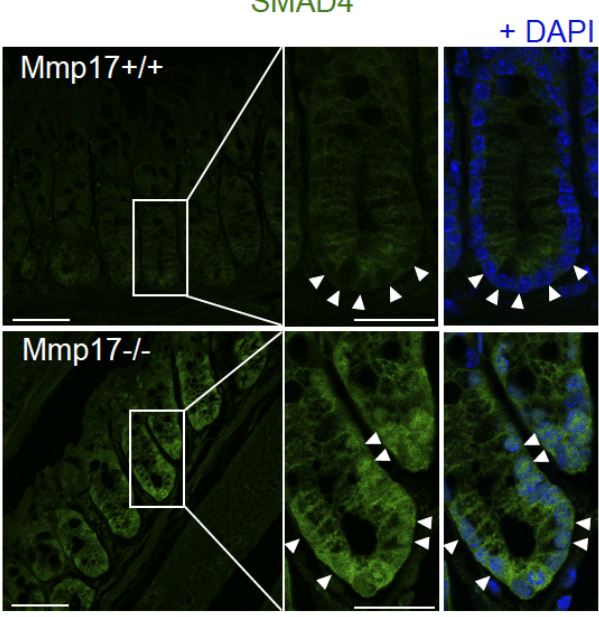

SMAD4 b

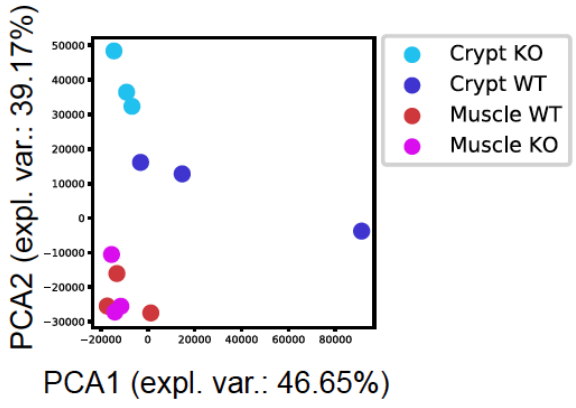

e

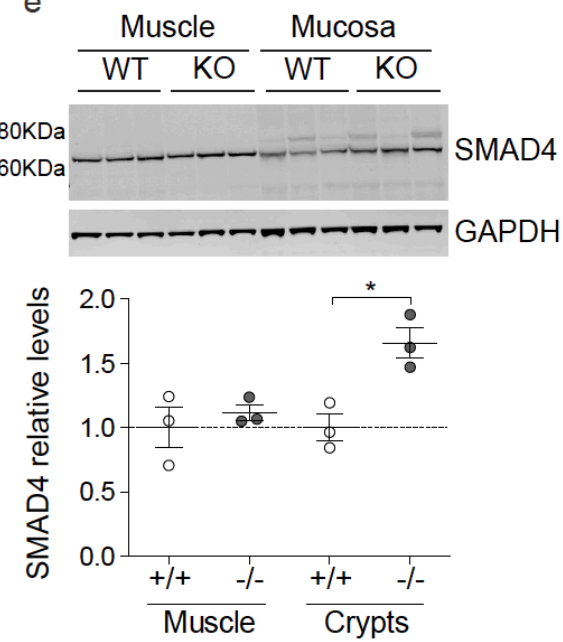

f

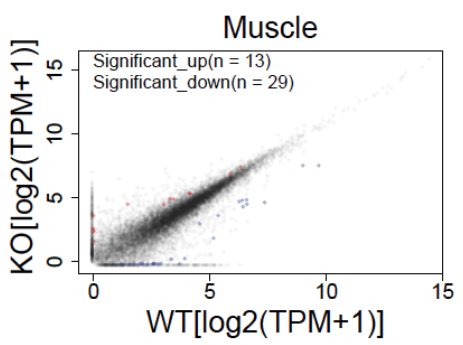

pSmad $1 / 5 / 9$

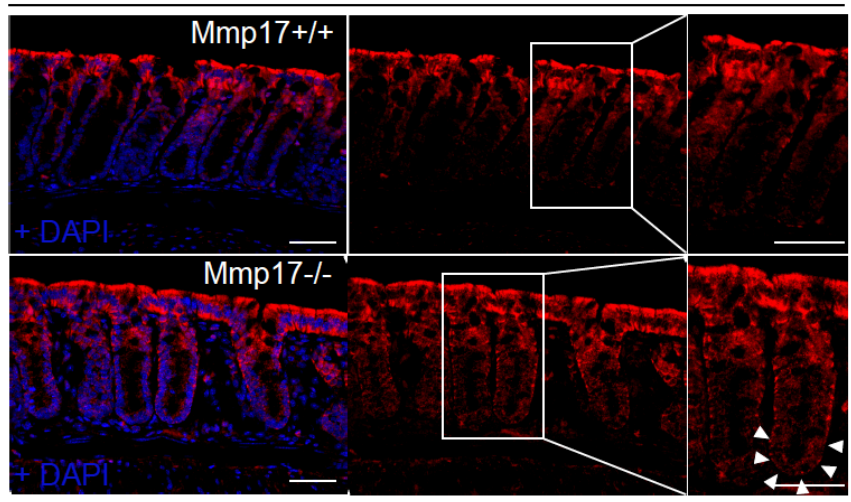

Figure 3: Muscle Mmp17 regulates crypts BMP signaling. a and b. RNA seq assay comparing WT and Mmp17 KO crypts, we showed that loss of MMP17 in the muscle strongly affects epithelial gene expression to a greater extent than muscle gene expression. $n=3$ biological replicates. c. Graph shows presence of genes related to SMAD4 signaling among the upregulated genes in WT vs KO crypts. $d$ and e We observed higher SMAD4 signaling that was further confirmed by immunofluorescence in $d$ (arrowheads indicate nuclear location in $M m p 17 \mathrm{KO}$ ) and western blot (e). $\mathrm{n}=3 \mathrm{mice} / \mathrm{genotype}$. Scale bar $50 \mu \mathrm{m}, 25 \mu \mathrm{m}$ in magnified views to the right. f. Representative confocal images showing pSmad 1/5/9 staining in intestinal crypts of WT vs KO mice.

Arrowheads highlight crypt base pSmad 1/5/9 staining. Scale $50 \mu \mathrm{m} . \mathrm{n}=2$ biological replicates. Numerical data in e are means $\pm S D$ and were tested by one-way ANOVA followed by Tukey's test.

\section{MMP17 regulates the ECM-ISC niche necessary for epithelial de novo crypt formation}


SMAD signaling is essential to maintain ISCs, which is exemplified by the requirement of the BMP antagonist NOGGIN in intestinal organoid maintenance (Sato et al. 2009). To test if MMP17 affects ISCs, we quantified levels of the ISC markers Lgr5 and Olfm4 by ISH. We found that KO intestinal tissue had lower Lgr5 and Olfm4 levels compared to WT tissue in both the small and large intestines (Fig. 4a, 4b). In addition, decreased ISC marker gene expression was echoed by reduced organoid formation efficiency comparing KO with WT crypts (Figure 4c and Figure S4b). Importantly, organoid splitting, or culturing with excessive niche factors, resulted in equal organoid efficiency between WT and KO cultures (Fig. 4d). These data indicate that the reduced capacity of KO crypts to form organoids relied on the in vivo niche rather than a consequence of an intrinsic epithelial defect.

a

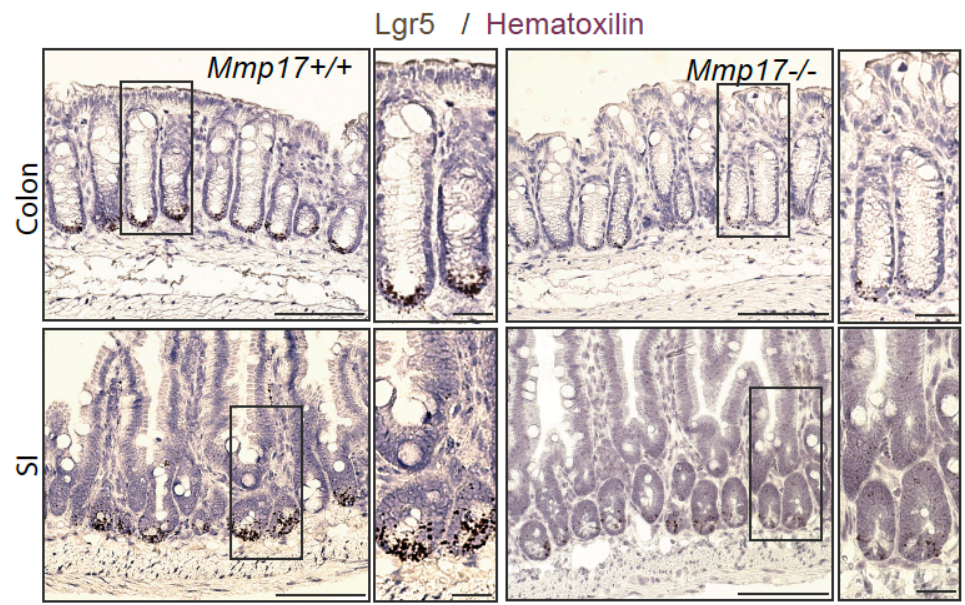

b

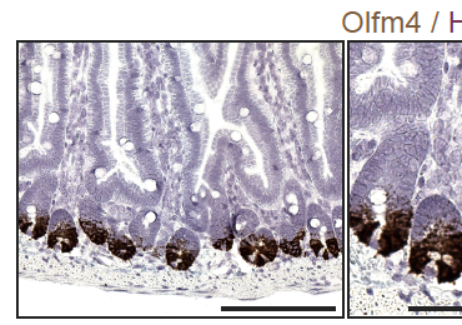

C
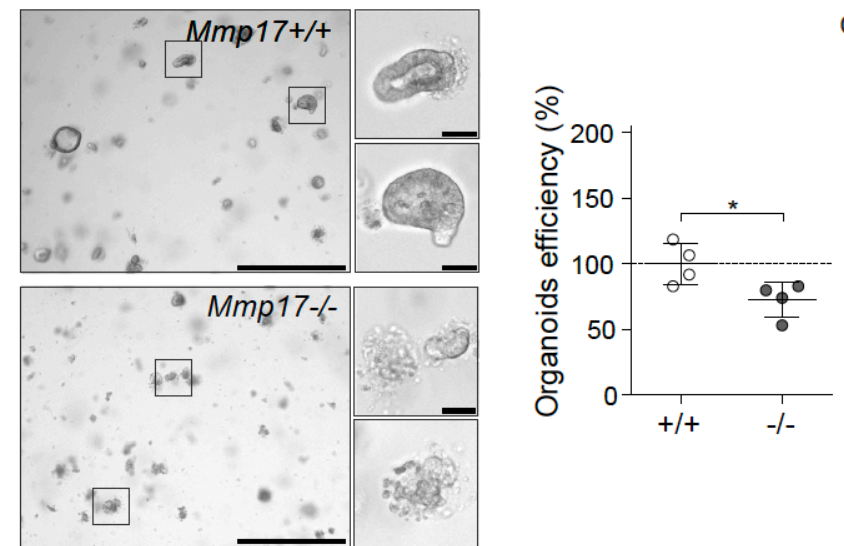
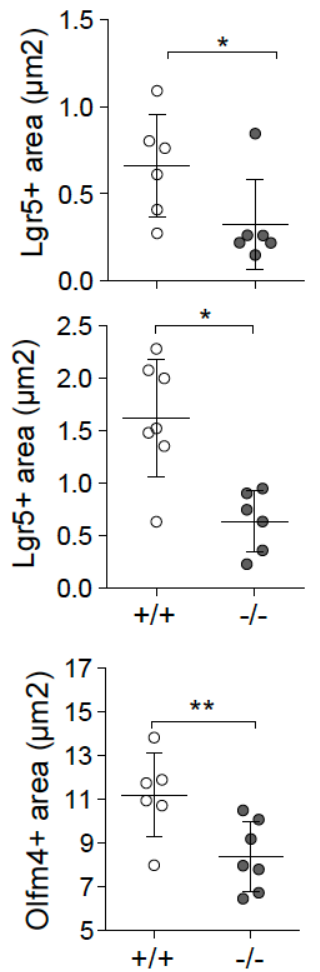
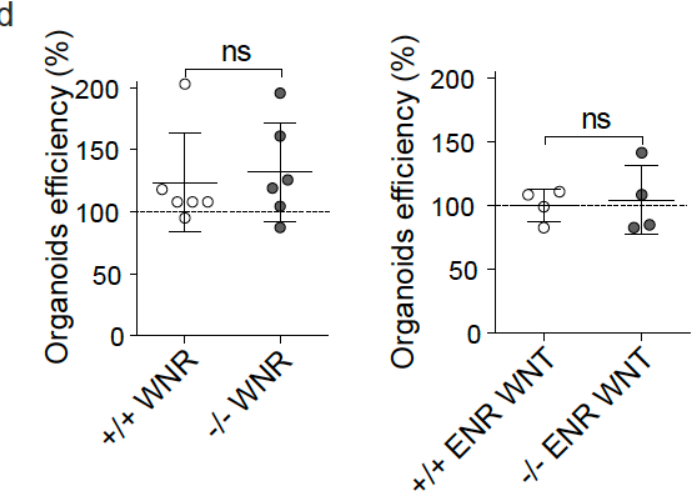

Figure 4. Muscular Mmp17 regulates crypt formation. a-b. Lgr5/Olfm4 RNAscope representative images of WT vs $\mathrm{KO}$ intestines and its quantification. Scale bar $100 \mu \mathrm{m} ; 25 \mu \mathrm{m}$ in inset. $\mathrm{n}=6$ mice per genotype analyzed in two different experiments. c. Representative bright field images of colon intestinal organoids from WT vs KO crypts $48 \mathrm{~h}$ 
after crypt isolation (left). Scale $650 \mu \mathrm{m} ; 50 \mu \mathrm{m}$ in cropped image. Graph shows relative organoids number as percentage normalized to WT. Each dot represents a well. One representative experiment is showed, out of 5 performed with similar results. d. Graphs represent the percentage of organoids derived from colon crypts after $72 \mathrm{~h}$ in response to the enriched medium WNR (left) and this percentage after splitting (right). Numerical data are means \pm SD and were tested by Mann-Whitney test.

\section{MMP17 is required for intestinal repair after inflammation or radiation-induced injury}

De novo crypt formation is important during epithelial repair which is needed upon injury, for example after damage inflicted by inflammation or due to irradiation. To test if smooth muscle, and in particular MMP17, could play a role in intestinal injury responses, we used Dextran Sulfate Sodium (DSS) to induce experimental colitis and compare WT to KO littermates (Fig. 5a). At day 5, we found that both WT and KO mice had indistinguishable features of disease indicating that damage was induced equally (Fig. S5a-e). After 5 days, DSS was replaced by regular drinking water to allow for intestinal repair, which is initiated rapidly and requires reprogramming of the intestinal epithelium (Yui et al. 2018; Miyoshi et al. 2017). Two days after DSS, KO mice had shorter colons and sustained hemorrhage, including an increased presence of blood in stool compared to WT mice (Fig. 5b, 5c). Furthermore, other disease features were also exacerbated in KO mice compared to WT mice. We found that KO mice had a higher injury score than WT mice, as was determined using a genotype-blind injury classification based on H\&E images (Fig. 5d, 5e). H\&E analysis further revealed a larger area of ulcerated mucosa and lower presence of epithelial crypts in KO mice compared to WT mice (Fig. 5e). In addition, we found a significant reduction in proliferative epithelium in KO mice (Fig. 5f), suggesting that epithelial reprogramming towards a reparative state relies on MMP17.

Next, we performed an alternative non-inflammatory injury model based on whole-body irradiation. Indeed, a single dose of ionizing radiation (10Gy) induces equal apoptosis in WT and KO intestines (Figure S5f). As custom in this model, WT animals regained crypt-villus structures 3 days after irradiation, however, small intestines of KO mice displayed disorganized epithelia with thicker and blunted villi (Figure $5 g$, S5g for quantification). In addition, a genotype-blinded evaluation of damage features in H\&E images showed increased signs of damage in small intestines of KO mice compared to WT mice (Figure 5h). Together, these data indicate that muscle-specific MMP17 is required for appropriate intestinal epithelial reparative responses upon injury. 

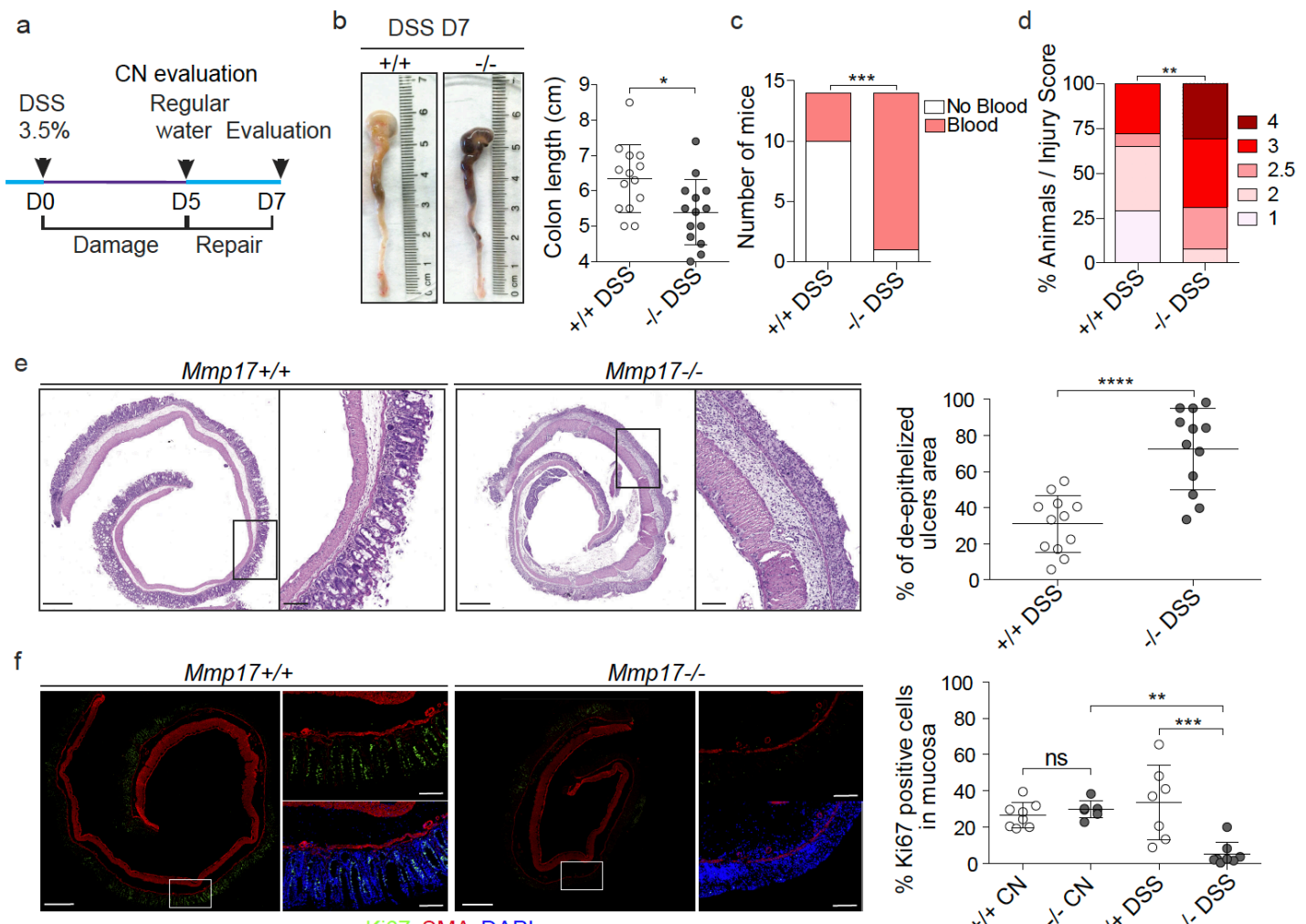

g

Ki67 SMA DAPI

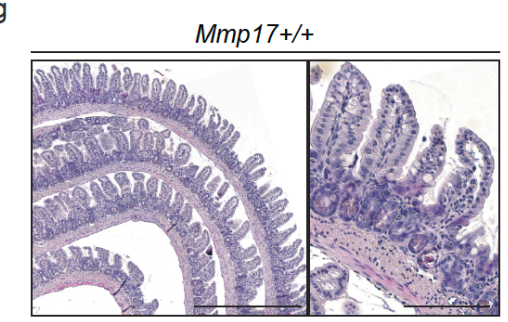

Mmp17-/-

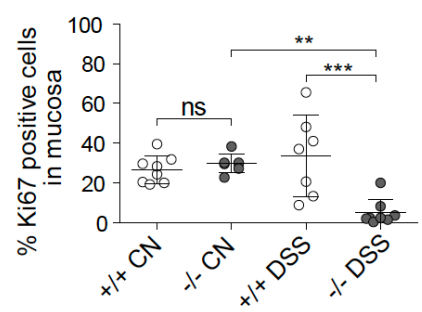

$\mathrm{h}$
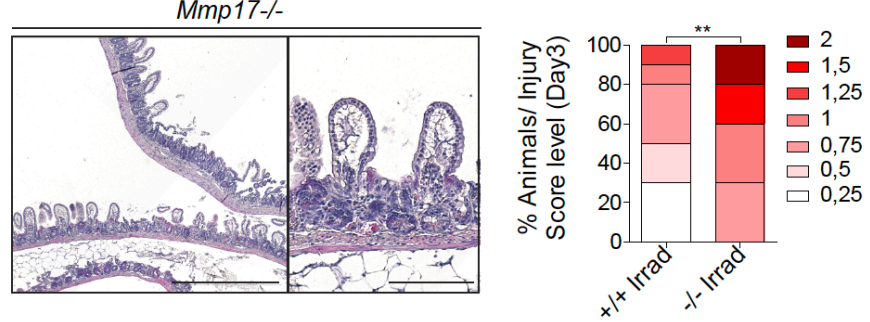
Figure 5. Muscle-specific Mmp17 is required for intestinal repair after injury. a. Timeline of DSS treatment. Mice were exposed to $3.5 \%$ DSS for 5 days followed by 2 days of regular water to allow epithelial restoration. Evaluation of control (CN) mice was performed at day 5 (D5). b. Representative images of mice colon in DSStreated animals at D7. Graph shows colon length, and presence of blood in stool and/or colonic cavity (right) at end point (D7) in c. $n=14$ animals per genotype, obtained from 5 independent experiments. d. Blind scoring of DSS-derived injury in colon, based on histological features. $n=14$ mice per genotype from 5 independent experiments. e. H\&E images showing distal colon in swiss roll. Scale $500 \mu \mathrm{m} ; 100 \mu \mathrm{m}$ in magnified views to the right. Graph represents the damaged mucosa as \% of area with ulcers relative to the total length of the distal colon. $n=12$ mice per genotype. f. Representative maximal projection of confocal images of DSS-treated distal colon stained for the proliferative marker Ki67 (Green), SMA (Red) and DAPI (Blue). Scale $500 \mu \mathrm{m} ; 100 \mu \mathrm{m}$ in magnified views. Graph shows \% of proliferative cells (Ki67 positive) in mucosa, normalized to total mucosal cell number. $n=7-8$ mice per genotype. g. Representative images of H\&E sections of small intestinal swiss rolls 3 days after 10Gy irradiation. Scale $500 \mu \mathrm{m} ; 100 \mu \mathrm{m}$ in magnified views. $n=7-8$ mice per genotype analyzed from 2 independent experiments. h. Blind scored injury level in irradiated mice. $n=7-8$. Numerical data in $b$, e and $f$ are represented as means \pm SD. Data in b and e were tested by t-test. Data in c was analyzed by Fisher's exact test (One-tailed) and in $\mathrm{d}$ and $\mathrm{h}$ were analyzed by Mann-Whitney test for nonparametric populations. Data in $\mathrm{f}$ was analyzed by one way ANOVA followed by Tukey's Multiple Comparison test.

Mmp17 loss results in long-term reparative epithelial defects and increased tumor risk We wondered whether intestines of KO mice would eventually heal, and thus we evaluated weight gain for a prolonged time after DSS administration (Fig. 6a, 6b). WT mice rapidly returned to their original weight; however, $\mathrm{KO}$ mice never fully regained their starting weight (Fig. 6b for females, Fig. S6a for males). In support, while WT mice largely returned to homeostatic conditions at end point, $\mathrm{KO}$ mice retained shorter colons, experienced sustained blood in stool, still had areas of unhealed ulcers in the epithelial surface and had higher injury score compared to WT mice (Fig. 6c-h). In addition, we detected the presence of crypt distortions named reactive atypia, and these morphological changes were predominantly found in KO mice (Fig. 6f, 6g, S6b). These morphological changes resemble those found in intestinal neoplastic lesions, so we next decided to evaluate the impact of Mmp17 loss in the initiation and progression of intestinal tumors using the $A p c^{\mathrm{Min}}$ mouse model. $A p c^{M i n}$ mice develop tumors primarily in the small intestinal epithelium (McCart, Vickaryous, and Silver 2008). We found that loss of Mmp17 predispose mice to the formation of a higher number of tumors both quantified macroscopically (Fig. 6i), and microscopically using H\&E stained sections (Figure 6j). We did not observe differences in tumor area (Fig. S6c) suggesting that MMP17 mediates tumor initiation but not tumor progression. In support, WT and KO tumors were indistinguishable in terms of $\beta$-CATENIN and OLFM4 distribution (Fig. S6d). Of note, Mmp17 expression was restricted to muscle cells also in tumor areas (Fig. S6e). In sum, our data indicate that MMP17 is required for short and long term intestinal epithelial repair and its loss predisposes to intestinal neoplastic 
a

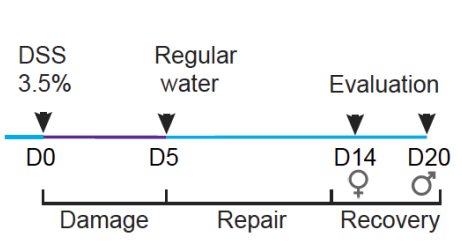

c

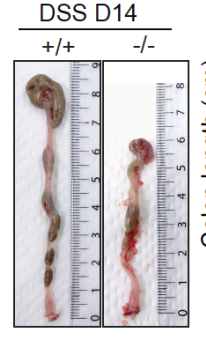

g

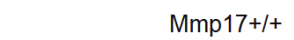

b

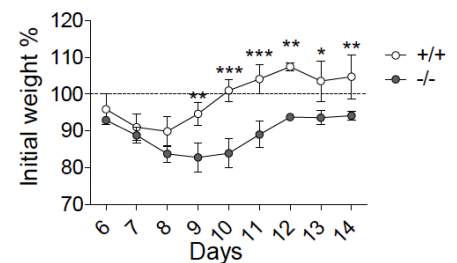

e

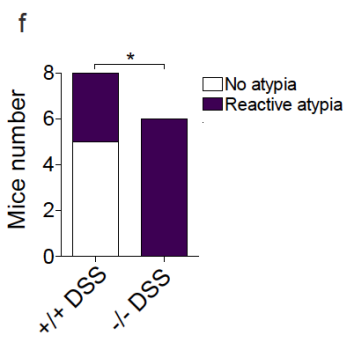

Mmp17-I-

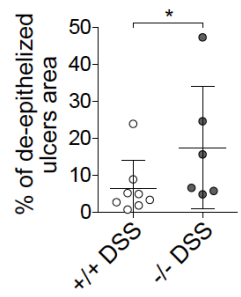

h
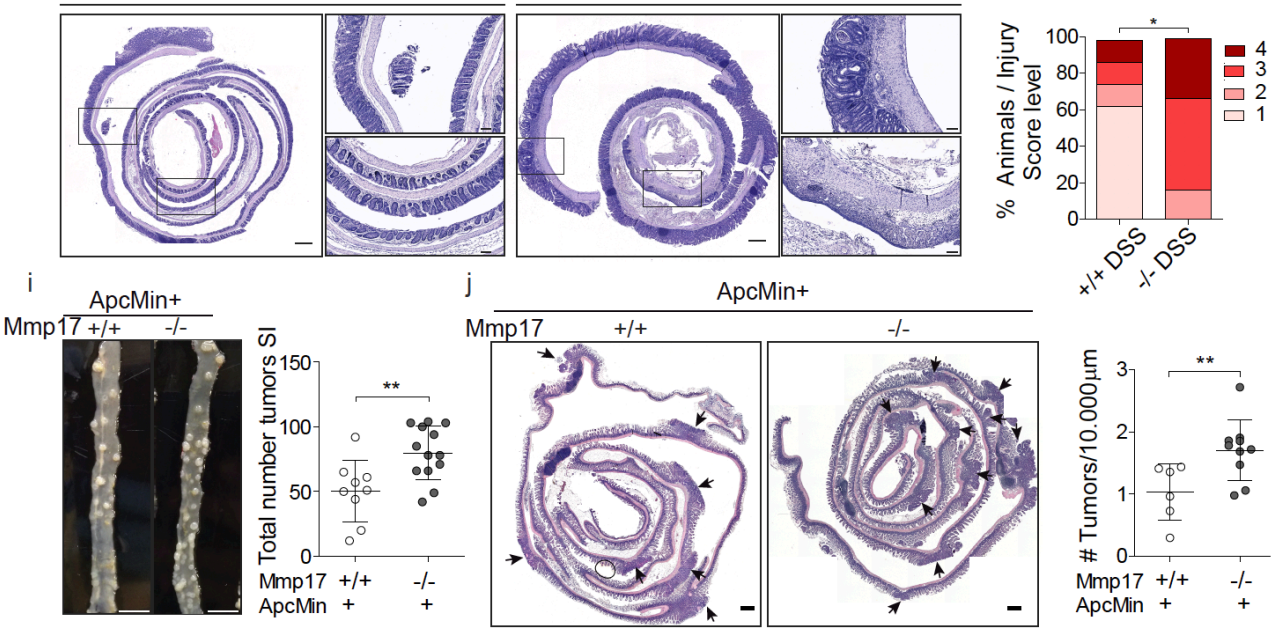

Figure 6. MMP17 absence hinders long-term repair in mouse intestinal epithelia and leads to increased tumorigenesis. a. Timeline of DSS-long treatment. Mice were exposed to $3.5 \%$ DSS for 5 days followed by 14 (females) or 19 days (males) days of regular water to allow epithelial restoration. b. Weight loss and recovery relative to $\%$ to initial weigh. $n=3$ to 4 mice per genotype and gender. Represented one experiment with females. c. Representative images showing colon length at end point and its quantification. $n=6-8$ mice. d. Graph shows presence of blood in stool/colon lumen at end point. $n=6-8$ mice per genotype. e. Graph represents the percentage of unhealed areas (ulcers) in WT and KO colonic mucosa at long term. f. Graph shows incidence of reactive atypia (or reactive epithelial changes after DSS). g. Representative H\&E pictures of colon swiss rolls showing healed crypts in WT vs ulcered areas and reactive atypia in KO. n= 6-8 mice per genotype. Scale $500 \mu \mathrm{m}$. h. Injury score representation of damaged colon evaluation. $n=6-8$ mice per genotype. i. Representative pictures showing tumor incidence in a portion of the small intestine of ApcMin+ Mmp17 WT and KO mice (Jejunum). Scale bar $1 \mathrm{~cm}$. Graph shows total number of tumors counted in fresh tissue (small intestine complete length). $n=9$ to 13 mice per genotype. j. H\&E representative images of small intestine swiss rolls in transverse cut. Arrows highlight visible tumors. Scale bar $500 \mu \mathrm{m}$. Graph shows tumor quantification normalized to tissue length. $n=6$ to 10 mice per genotype. Numerical data in $b$ was analyzed by two way ANOVA followed by Bonferroni posttest. $t$ test (normal distribution) was applied in c. Fisher's exact test, one tailed was used in $\mathrm{d}$ and f. Numerical data in e and $\mathrm{h}$ were analyzed by Mann-Whitney t-test. Data in i, $\mathrm{j}$ were analyzed by unpaired t-test. 
Since we observed that smooth muscle MMP17 is essential for intestinal repair we wondered whether muscle indeed can promote epithelial reprogramming towards a reparative state. We found that muscle-SN can replace NOGGIN in the culture media, and muscle-SN treated organoids grow as large spheroids (Fig. 1). Organoid spheroid growth can either be characterized as reparative that is associated with fetal-like gene programs, such as organoids derived from SCA-1+ cells (Nusse et al. 2018), or it can be the result of increased WNT signalling such as upon treatment with the GSK3 inhibitor CHIR (Yin et al. 2014). These two different organoid spheroid states are on opposite ends when it concerns ISCs; a reparative state is characterized by a loss of LGR5+ ISCs whereas ISCs expand upon CHIR treatment (Yui et al. 2018; Nusse et al. 2018). To determine what type of spheroids are induced by muscle-SN, we performed RNA-seq comparing normal organoids to muscle-SN treated organoids (Fig. 7a). Using gene set enrichment analysis, we found that genes associated with LGR5+ cells, as well as genes upregulated in organoids treated with $\mathrm{CHIR}$, were downregulated in muscle-SN treated organoids (Fig. 7b). In contrast, gene sets associated with repair and fetal programs were significantly enriched in muscle-SN treated organoids (Fig. 7b). Intestinal epithelial repair programs are coupled with YAP signalling and, indeed, a YAP signature gene set was also enriched in muscle-SN treated organoids (Fig. 7b). In support, we found that YAP was localized nuclear throughout muscle-SN induced spheroids, whereas it was cytoplasmic in the centre of budding organoids (Fig. 7c). Together, we conclude that muscle-SN induces spheroid growth that is associated with reparative/fetal-like reprogramming and may be mediated by YAP.

\section{PERIOSTIN is a muscle-derived factor cleaved by MMP17 that activates YAP and induces organoid growth}

MMP17 is expressed in intestinal smooth muscle and is required to allow for epithelial repair in vivo (Figs. 5, 6). Furthermore, muscle-SN is able to reprogram epithelium towards a reparative state in vitro (Fig. 7a-c). We therefore hypothesize that MMP17 cleaves a musclederived factor that facilitates epithelial reprogramming that is needed for repair. To identify said factor, we examined muscle-derived proteins by performing mass-spectrometry on muscle-SN, and we detected 550 proteins (Suppl. Table 1). We next curated this list to only display proteins known to be growth/niche factors and/or extracellular proteins (Fig. 7d). Among these was PERIOSTIN (POSTN), which is a matricellular protein that is highly expressed in smooth muscle (Fig. 7e). We previously identified a different matricellular protein, OSTEOPONTIN (OPN), as an MMP17 substrate (Martín-Alonso et al. 2015). Of note, POSTN can serve as a ligand for integrins to activate YAP signalling (Ma et al. 2020). Further, POSTN has been proposed to capture BMP members in the ECM (Maruhashi et al. 2010). Both of these features are relevant for biological processes we identified to be 

indicating MMP17 is able to cleave POSTN (Fig. 7f). In addition, we examined POSTN in MMP17-WT and KO smooth muscle and we found a decrease in POSTN fragments in KO muscle samples, suggesting that cleavage of POSTN also occurs in vivo and it is impaired in KO intestines (Fig. 7g). Finally, we found that POSTN itself induces organoid growth that is associated with induction of Ki67 and nuclear YAP (Fig. 7h-j).

a
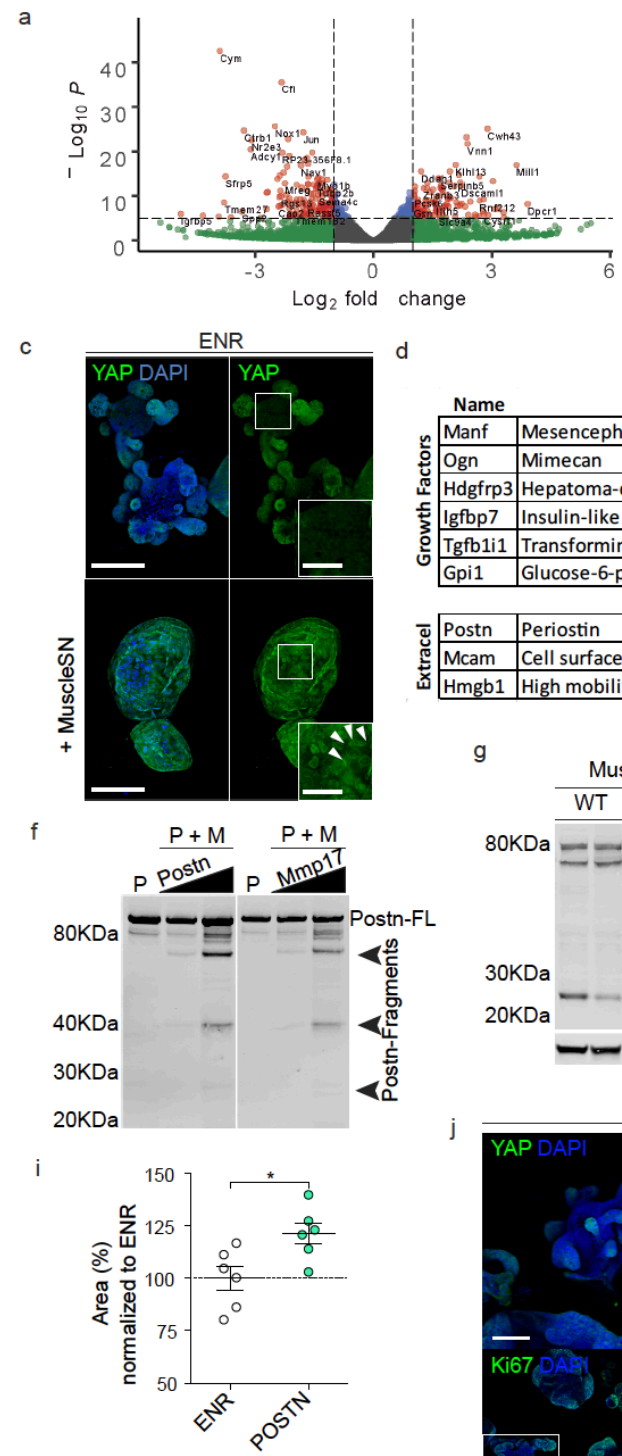

g
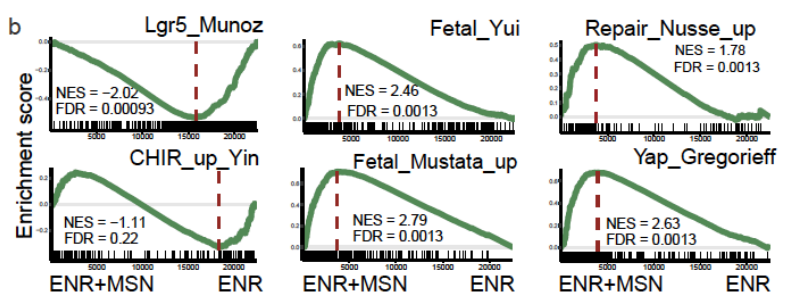

e
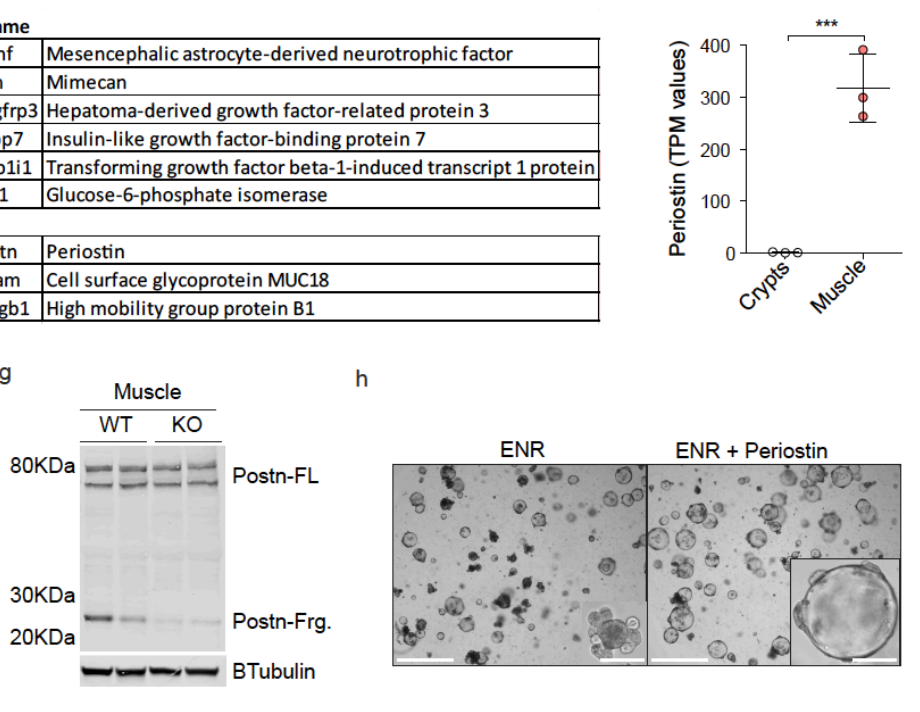

$\mathrm{h}$
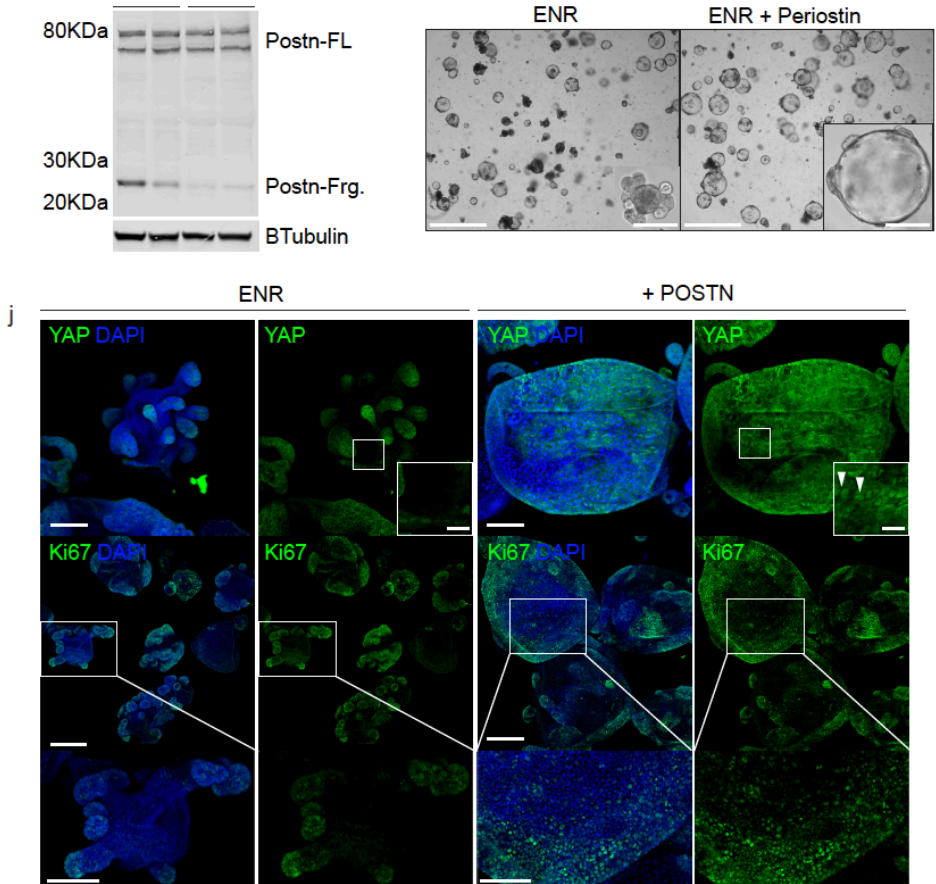

+ POSTN

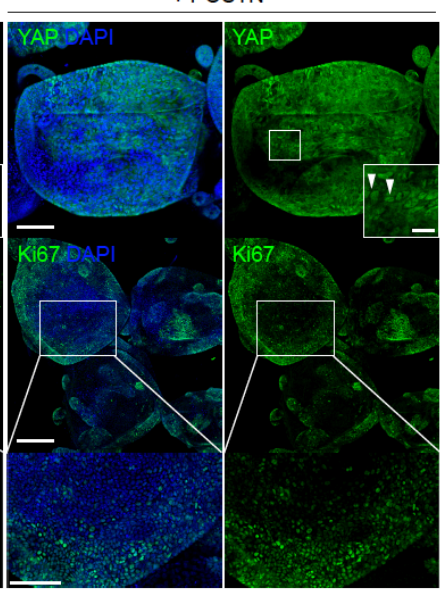

Figure 7. Identification of muscle-derived factor PERIOSTIN as an in vivo and in vitro substrate for MMP17. a. Volcano plot showing significantly up and downregulated genes in organoids exposed to muscle-SN vs ENR. b. GSEA of indicated gene sets comparing ENR with MuscleSN treated organoids. NES (normalized enrichment score) and FDR (false discovery rate) are depicted. c. Representative maximal projection confocal images showing cytoplasmic vs nuclear YAP staining (green). Scale $100 \mu \mathrm{m} ; 25 \mu \mathrm{m}$ in inset. Arrowheads 
highlight nuclear YAP. $n=2$ independent experiments. $d$. List of growth factors and extracellular proteins found in muscle-SN. $n=3$ biological replicates. e. TPM values for Postn comparing crypts with smooth muscle tissue. $n=3$ biological replicates. $\mathrm{f}$. In vitro digestion experiment with human recombinant proteins showing POSTN fragments when in contact with MMP17 catalytic domain. P, POSTN, M, MMP17, FL, full length. $n=2$ experiments with increasing concentrations of POSTN or MMP17. g. WB of mouse intestinal muscle showing decreased POSTN fragments in the absence of MMP17. $n=2$ biological replicates. $h$. Representative brightfield images showing organoids morphology in the presence of POSTN and area quantification (Day 3) (i). Scale 1250 and $200 \mu \mathrm{m}$ in inset. $n=3$ independently performed experiments with 2-3 wells/experiment. j. Representative confocal maximum intensity projection images showing YAP and Ki67 (green) staining in POSTN-treated organoids. Scale $100 \mu \mathrm{m}$; $25 \mu \mathrm{m}$ in inset (YAP pictures) and $200 \mu \mathrm{m}$ and $100 \mu \mathrm{m}$ in Ki67 pictures. Arrows highlight nuclear YAP. Numerical data are means \pm SD. Data in e represents $p$-adjusted value from RNAseq analysis and data in $i$ was analyzed by Mann-Whitney test.

\section{DISCUSSION}

The role of smooth muscle cells, other than in peristalsis, has been largely undefined. However, in a recent preprint, it was found that in early human gut development ACTA2+ muscularis mucosa cells are the major source of WNT, RSPO, and GREM niche factors (Czerwinski et al. 2020). In contrast, in adult mice, there have been various mucosa-resident mesenchymal cell populations described that express Rspo and Wnt genes (Greicius et al. 2018a; Shoshkes-Carmel et al. 2018b; Degirmenci et al. 2018). We here find that smooth muscle, and in particular, the muscularis mucosa, is the primary source of BMP antagonists such as Grem1/2 (Fig. 1). The importance of GREMLIN1 as an ICS niche factor was recently determined by an experiment in which Grem1-expressing cells were depleted using the diphtheria-toxin system, which also led to the rapid loss of LGR5+ ISCs (McCarthy et al. 2020). This experiment would thus have led to the death of practically all muscularis-mucosa smooth muscle cells. Together, our work here as well as recent work by others highlight the importance of smooth muscle for providing niche factors important for ISC homeostasis.

We find an important role for MMP17 in vivo. We show that KO mice have a reduction of ISC-associated genes in homeostasis (Fig. 4), which may be caused by increased levels of SMAD4 at the bottom of KO crypts (Fig. 3). Indeed, BMP signalling can impair ISC-signature genes by direct repression via SMAD1/4 recruitment of HDAC1 (Qi et al. 2017). We further identify that MMP17 is required for intestinal epithelial repair after injury, which we link to the ability of MMP17 to cleave POSTN. We hypothesize that MMP17 cleavage of POSTN is necessary upon injury to reprogram epithelial cells in a YAP-dependent manner. Earlier this year, Ma et al. described a role for POSTN in activating YAP/TAZ through an integrin-FAKSrc pathway using cancer cell lines (Ma et al. 2020). We here extend these findings and show that POSTN can also affect primary non-tumor cells (Fig. 7). In addition to this direct role affecting the epithelium, others have shown that POSTN can alter the ECM by binding 
to a variety of ECM associated proteins including BMP1, FIBRONECTIN, and TENASCIN-C (Kudo and Kii 2018). We would speculate that through this link with the ECM, cleaved POSTN may broadly affect intestinal homeostasis in vivo, which would be impaired in MMP17-deficient mice such as we observe.

In addition, others have also shown that POSTN is cleaved in vivo, suggesting this has a physiological role, and Postn-deficient mice have general issues with repair in various tissues (Shimazaki et al. 2008; Nishiyama et al. 2011). Thus, although the importance of cleavage of POSTN remains not fully comprehended (Kudo 2011), it is clear that POSTN plays an important role in reparative processes and the identification of MMP17 as an enzyme that can cleave POSTN can be used in future studies.

MMP17, unlike other MMP members, exert minimal activity against classical ECM components (Kolkenbrock et al. 1999). So far, only a few bona fide substrates have been described in other tissue environments, such as ADAMTS-4 (Clements et al. 2011), alphaM integrin (Clemente et al. 2018) and two matricellular proteins; OPN (Martín-Alonso et al. 2015) and POSTN described in this work. We can not discard the possibility that these or other unidentified MMP17 substrates play a role in ISCs niche regulation or the intestinal response to injury, particularly OPN which expression is increased in patients with inflammatory bowel disease (Neuman 2012). Furthermore, in a tumor environment, both ADAMTS4 and OPN are overexpressed in colon cancers (M. Zhao et al. 2015; H. Zhao et al. 2018; J. Chen et al. 2018). Finally, also non-catalytic activities for MMP17 have been described related to tumors (Paye et al. 2014).

To summarize, we discover a previously unappreciated role for intestinal smooth muscle tissue. We find that smooth muscle are likely the major contributor of BMP antagonists, which are essential niche factors for the maintenance of ISCs. In addition, we describe an important role for smooth-muscle restricted expression of MMP17, which in vivo is required for epithelial repair. Finally, we provide evidence that MMP17 acts via cleavage of the matricellular protein POSTN, which in itself can induce repair-like features in intestinal epithelium.

\section{ACKNOWLEDGEMENTS}

We would like to thank Dr. Motoharu Seiki for kindly sharing the $M m p 17^{\mathrm{KO} / \mathrm{KI}}$ mouse line and Dr. Kaisa Lehti for first inspiring this study. We would like to thank to Anne Beate L. Marthinsen for performing the irradiation, and the Department of Radiology and Nuclear Medicine (St. Olav's Hospital) for allowing the use of their instruments. We also thank Arne Wibe and Elin Rønne for their evaluation of colon crypt reactive atypia. We thank the 
imaging (CMIC) and animal care (CoMed) core facilities (NTNU), as well as the histology and animal facilities at CNIC for assisting in this work. The WT KO crypt-muscle RNA-seq was done by the Genomics Core Facility at NTNU, which receives funding from the Faculty of Medicine and Health Sciences and Central Norway Regional Health Authority. This work was further financially supported by the Norwegian Research Council (Centre of Excellence grant 223255/F50, and 'Young Research Talent' 274760 to MJO) and the Norwegian Cancer Society (182767 to MJO). MMA is the recipient of a Marie Skłodowska-Curie IF (DLV794391).

\section{DATA DEPOSITION}

All raw sequencing data is available through ArrayExpress: WT and KO smooth muscle and crypt RNA seq: E-MTAB-9180 ;ENR vs MuscleSN treated organoids RNA seq: EMTAB9181.

\section{REFERENCES}

Barker, Nick, Johan H. Van Es, Jeroen Kuipers, Pekka Kujala, Maaike Van Den Born, Miranda Cozijnsen, Andrea Haegebarth, et al. 2007. "Identification of Stem Cells in Small Intestine and Colon by Marker Gene Lgr5." Nature. https://doi.org/10.1038/nature06196.

Chen, Edward Y., Christopher M. Tan, Yan Kou, Qiaonan Duan, Zichen Wang, Gabriela V. Meirelles, Neil R. Clark, and Avi Ma'ayan. 2013. "Enrichr: Interactive and Collaborative HTML5 Gene List Enrichment Analysis Tool." BMC Bioinformatics. https://doi.org/10.1186/1471-2105-14-128.

Chen, Jianjun, Yang Luo, Yong Zhou, Shaolan Qin, Yier Qiu, Ran Cui, Minhao Yu, Jun Qin, and Ming Zhong. 2018. "Promotion of Tumor Growth by ADAMTS4 in Colorectal Cancer: Focused on Macrophages." Cellular Physiology and Biochemistry. https://doi.org/10.1159/000489245.

Clemente, Cristina, Cristina Rius, Laura Alonso-Herranz, Mara Martín-Alonso, Ángela Pollán, Emilio Camafeita, Fernando Martínez, et al. 2018. "MT4-MMP Deficiency Increases Patrolling Monocyte Recruitment to Early Lesions and Accelerates Atherosclerosis." Nature Communications 9 (1): 4-6. https://doi.org/10.1038/s41467018-03351-4.

Clements, Kristen M., Jo K. Flannelly, Jonathan Tart, Sarah M.V. Brockbank, John Wardale, Jim Freeth, Andrew E. Parker, and Peter Newham. 2011. "Matrix Metalloproteinase 17 Is Necessary for Cartilage Aggrecan Degradation in an Inflammatory Environment." Annals of the Rheumatic Diseases. https://doi.org/10.1136/ard.2010.130757. 
Katherine D Walton, et al. 2020. "In Vitro and in Vivo Development of the Human Intestinal Niche at Single Cell Resolution." BioRxiv, January, 2020.01.31.928788. https://doi.org/10.1101/2020.01.31.928788.

Degirmenci, Bahar, Tomas Valenta, Slavica Dimitrieva, George Hausmann, and Konrad Basler. 2018. "GLI1-Expressing Mesenchymal Cells Form the Essential Wnt-Secreting Niche for Colon Stem Cells." Nature 558 (7710): 449-53. https://doi.org/10.1038/s41586-018-0190-3.

Esteban, Sergio, Cristina Clemente, Agnieszka Koziol, Pilar Gonzalo, Cristina Rius, Fernando Martínez, Pablo M Linares, et al. 2020. “ Endothelial MT 1- MMP Targeting Limits Intussusceptive Angiogenesis and Colitis via TSP1/Nitric Oxide Axis ." EMBO Molecular Medicine. https://doi.org/10.15252/emmm.201910862.

Gattazzo, Francesca, Anna Urciuolo, and Paolo Bonaldo. 2014. "Extracellular Matrix: A Dynamic Microenvironment for Stem Cell Niche." Biochimica et Biophysica Acta General Subjects. https://doi.org/10.1016/j.bbagen.2014.01.010.

Gjorevski, Nikolce, Norman Sachs, Andrea Manfrin, Sonja Giger, Maiia E. Bragina, Paloma Ordóñez-Morán, Hans Clevers, and Matthias P. Lutolf. 2016. "Designer Matrices for Intestinal Stem Cell and Organoid Culture." Nature.

https://doi.org/10.1038/nature20168.

Gregorieff, Alex, Yu Liu, Mohammad R. Inanlou, Yuliya Khomchuk, and Jeffrey L. Wrana. 2015. "Yap-Dependent Reprogramming of Lgr5+ Stem Cells Drives Intestinal Regeneration and Cancer." Nature. https://doi.org/10.1038/nature15382.

Greicius, Gediminas, Zahra Kabiri, Kristmundur Sigmundsson, Chao Liang, Ralph Bunte, Manvendra K. Singh, and David M. Virshup. 2018a. "PDGFRa+ Pericryptal Stromal Cells Are the Critical Source of Wnts and RSPO3 for Murine Intestinal Stem Cells in Vivo." Proceedings of the National Academy of Sciences of the United States of America. https://doi.org/10.1073/pnas.1713510115.

- 2018b. "PDGFR $\alpha+$ Pericryptal Stromal Cells Are the Critical Source of Wnts and RSPO3 for Murine Intestinal Stem Cells in Vivo." Proceedings of the National Academy of Sciences of the United States of America 115 (14): E3173-81. https://doi.org/10.1073/pnas.1713510115. Jakubowska, Katarzyna, Anna Pryczynicz, Piotr Iwanowicz, Andrzej Niewiński, Elzbieta Maciorkowska, Jerzy Hapanowicz, Dorota Jagodzińska, Andrzej Kemona, and Katarzyna Guzińska-Ustymowicz. 2016. "Expressions of Matrix Metalloproteinases (MMP-2, MMP-7, and MMP-9) and Their Inhibitors (TIMP-1, TIMP-2) in Inflammatory Bowel Diseases." Gastroenterology Research and Practice. https://doi.org/10.1155/2016/2456179. 

in Mesenchymal Cells Required for Suppression of Gastrointestinal Polyposis." Nature Genetics. https://doi.org/10.1038/ng.98.

Kessenbrock, Kai, Chih Yang Wang, and Zena Werb. 2015. "Matrix Metalloproteinases in Stem Cell Regulation and Cancer." Matrix Biology. https://doi.org/10.1016/j.matbio.2015.01.022.

Kolkenbrock, Hansjörg, Lutz Essers, Norbert Ulbrich, and Horst Will. 1999. "Biochemical Characterization of the Catalytic Domain of Membrane-Type 4 Matrix Metalloproteinase." Biological Chemistry. https://doi.org/10.1515/BC.1999.137.

Kudo, Akira. 2011. "Periostin in Fibrillogenesis for Tissue Regeneration: Periostin Actions inside and Outside the Cell." Cellular and Molecular Life Sciences 68 (19): 3201-7. https://doi.org/10.1007/s00018-011-0784-5.

Kudo, Akira, and Isao Kii. 2018. "Periostin Function in Communication with Extracellular Matrices." Journal of Cell Communication and Signaling. https://doi.org/10.1007/s12079-017-0422-6.

Kuleshov, Maxim V., Matthew R. Jones, Andrew D. Rouillard, Nicolas F. Fernandez, Qiaonan Duan, Zichen Wang, Simon Koplev, et al. 2016. "Enrichr: A Comprehensive Gene Set Enrichment Analysis Web Server 2016 Update." Nucleic Acids Research. https://doi.org/10.1093/nar/gkw377.

Ma, Handong, Jing Wang, Xueli Zhao, Tiantian Wu, Zhengjie Huang, Dafan Chen, Yingfu Liu, and Gaoliang Ouyang. 2020. "Periostin Promotes Colorectal Tumorigenesis through Integrin-FAK-Src Pathway-Mediated YAP/TAZ Activation." Cell Reports. https://doi.org/10.1016/j.celrep.2019.12.075.

Martín-Alonso, Mara, Ana B. García-Redondo, Dongchuan Guo, Emilio Camafeita, Fernando Martínez, Arántzazu Alfranca, Nerea Méndez-Barbero, et al. 2015. "Deficiency of MMP17/MT4-MMP Proteolytic Activity Predisposes to Aortic Aneurysm in Mice." Circulation Research. https://doi.org/10.1161/CIRCRESAHA.117.305108.

Maruhashi, Takumi, Isao Kii, Mitsuru Saito, and Akira Kudo. 2010. "Interaction between Periostin and BMP-1 Promotes Proteolytic Activation of Lysyl Oxidase." Journal of Biological Chemistry. https://doi.org/10.1074/jbc.M109.088864.

Massagué, Joan. 2012. "TGF $\beta$ Signalling in Context." Nature Reviews Molecular Cell Biology. https://doi.org/10.1038/nrm3434.

McCart, Amy E., Nicola K. Vickaryous, and Andrew Silver. 2008. "Apc Mice: Models, Modifiers and Mutants." Pathology Research and Practice 204 (7): 479-90. https://doi.org/10.1016/j.prp.2008.03.004.

McCarthy, Neil, Elisa Manieri, Elaine E. Storm, Assieh Saadatpour, Adrienne M. Luoma, Varun N. Kapoor, Shariq Madha, et al. 2020. “Distinct Mesenchymal Cell Populations 
Generate the Essential Intestinal BMP Signaling Gradient." Cell Stem Cell. https://doi.org/10.1016/j.stem.2020.01.008.

Meran, Laween, Anna Baulies, and Vivian S.W. Li. 2017. "Intestinal Stem Cell Niche: The Extracellular Matrix and Cellular Components." Stem Cells International 2017. https://doi.org/10.1155/2017/7970385.

Miyoshi, Hiroyuki, Kelli L VanDussen, Nicole P Malvin, Stacy H Ryu, Yi Wang, Naomi M Sonnek, Chin-Wen Lai, and Thaddeus S Stappenbeck. 2017. "Prostaglandin E2 Promotes Intestinal Repair through an Adaptive Cellular Response of the Epithelium." The EMBO Journal. https://doi.org/10.15252/embj.201694660.

Murata, Kazutaka, Unmesh Jadhav, Shariq Madha, Johan van Es, Justin Dean, Alessia Cavazza, Kai Wucherpfennig, Franziska Michor, Hans Clevers, and Ramesh A. Shivdasani. 2020. "Ascl2-Dependent Cell Dedifferentiation Drives Regeneration of Ablated Intestinal Stem Cells." Cell Stem Cell. https://doi.org/10.1016/j.stem.2019.12.011.

Neuman, Manuela G. 2012. "Osteopontin Biomarker in Inflammatory Bowel Disease, Animal Models and Target for Drug Discovery." Digestive Diseases and Sciences. https://doi.org/10.1007/s10620-012-2120-9.

Nishiyama, Takashi, Isao Kii, Takeshi G. Kashima, Yoshinao Kikuchi, Atsushi Ohazama, Masashi Shimazaki, Masashi Fukayama, and Akira Kudo. 2011. "Delayed ReEpithelialization in Periostin-Deficient Mice during Cutaneous Wound Healing." PLoS ONE 6 (4). https://doi.org/10.1371/journal.pone.0018410.

Nusse, Ysbrand M., Adam K. Savage, Pauline Marangoni, Axel K.M. Rosendahl-Huber, Tyler A. Landman, Frederic J. De Sauvage, Richard M. Locksley, and Ophir D. Klein. 2018. "Parasitic Helminths Induce Fetal-like Reversion in the Intestinal Stem Cell Niche." Nature 559 (7712): 109-13. https://doi.org/10.1038/s41586-018-0257-1. O'Sullivan, Shane, John F. Gilmer, and Carlos Medina. 2015. "Matrix Metalloproteinases in Inflammatory Bowel Disease: An Update." Mediators of Inflammation 2015. https://doi.org/10.1155/2015/964131.

Page-McCaw, Andrea, Andrew J. Ewald, and Zena Werb. 2007a. "Matrix Metalloproteinases and the Regulation of Tissue Remodelling." Nature Reviews Molecular Cell Biology. https://doi.org/10.1038/nrm2125.

_ 2007b. "Matrix Metalloproteinases and the Regulation of Tissue Remodelling." Nature Reviews Molecular Cell Biology 8 (3): 221-33. https://doi.org/10.1038/nrm2125. Paye, Alexra, Alice Truong, Cassre Yip, Jonathan Cimino, Silvia Blacher, Carine Munaut, Didier Cataldo, et al. 2014. "EGFR Activation and Signaling in Cancer Cells Are Enhanced by the Membrane-Bound Metalloprotease MT4-MMP." Cancer Research. https://doi.org/10.1158/0008-5472.CAN-13-2994. 
Qi, Zhen, Yehua Li, Bing Zhao, Chi Xu, Yuan Liu, Haonan Li, Bingjie Zhang, et al. 2017. "BMP Restricts Stemness of Intestinal Lgr5 + Stem Cells by Directly Suppressing Their Signature Genes." Nature Communications 8: 1-14.

https://doi.org/10.1038/ncomms13824.

Rikimaru, Akiko, Kiyoshi Komori, Takeharu Sakamoto, Hirotake Ichise, Nobuaki Yoshida, Ikuo Yana, and Motoharu Seiki. 2007. "Establishment of an MT4-MMP-Deficient Mouse Strain Representing an Efficient Tracking System for MT4-MMP/MMP-17 Expression in Vivo Using $\beta$-Galactosidase." Genes to Cells. https://doi.org/10.1111/j.13652443.2007.01110.x.

Rodríguez, David, Charlotte J. Morrison, and Christopher M. Overall. 2010. "Matrix Metalloproteinases: What Do They Not Do? New Substrates and Biological Roles Identified by Murine Models and Proteomics." Biochimica et Biophysica Acta Molecular Cell Research. https://doi.org/10.1016/j.bbamcr.2009.09.015.

Roulis, Manolis, Aimilios Kaklamanos, Marina Schernthanner, Piotr Bielecki, Jun Zhao, Eleanna Kaffe, Laura Sophie Frommelt, et al. 2020. "Paracrine Orchestration of Intestinal Tumorigenesis by a Mesenchymal Niche." Nature. https://doi.org/10.1038/s41586-020-2166-3.

Sato, Toshiro, Robert G. Vries, Hugo J. Snippert, Marc Van De Wetering, Nick Barker, Daniel E. Stange, Johan H. Van Es, et al. 2009. "Single Lgr5 Stem Cells Build CryptVillus Structures in Vitro without a Mesenchymal Niche." Nature. https://doi.org/10.1038/nature07935.

Serra, Denise, Urs Mayr, Andrea Boni, llya Lukonin, Markus Rempfler, Ludivine Challet Meylan, Michael B. Stadler, et al. 2019. "Self-Organization and Symmetry Breaking in Intestinal Organoid Development." Nature. https://doi.org/10.1038/s41586-019-1146-y. Shimazaki, Masashi, Kazuto Nakamura, Isao Kii, Takeshi Kashima, Norio Amizuka, Minqi Li, Mitsuru Saito, et al. 2008. "Periostin Is Essential for Cardiac Healing after Acute Myocardial Infarction." Journal of Experimental Medicine 205 (2): 295-303. https://doi.org/10.1084/jem.20071297.

Shoshkes-Carmel, Michal, Yue J. Wang, Kirk J. Wangensteen, Beáta Tóth, Ayano Kondo, Efi E. Massassa, Shalev Itzkovitz, and Klaus H. Kaestner. 2018a. "Subepithelial Telocytes Are an Important Source of Wnts That Supports Intestinal Crypts." Nature 557 (7704): 242-46. https://doi.org/10.1038/s41586-018-0084-4. _ 2018b. "Subepithelial Telocytes Are an Important Source of Wnts That Supports Intestinal Crypts." Nature. https://doi.org/10.1038/s41586-018-0084-4.

Tian, Hua, Brian Biehs, Søren Warming, Kevin G. Leong, Linda Rangell, Ophir D. Klein, and Frederic J. De Sauvage. 2011. "A Reserve Stem Cell Population in Small Intestine Renders Lgr5-Positive Cells Dispensable." Nature. https://doi.org/10.1038/nature10408. 
Wang, Yi, I. Ling Chiang, Takahiro E. Ohara, Satoru Fujii, Jiye Cheng, Brian D. Muegge, Aaron Ver Heul, et al. 2019. "Long-Term Culture Captures Injury-Repair Cycles of Colonic Stem Cells." Cell. https://doi.org/10.1016/j.cell.2019.10.015.

Yin, Xiaolei, Henner F. Farin, Johan H. Van Es, Hans Clevers, Robert Langer, and Jeffrey M. Karp. 2014. "Niche-Independent High-Purity Cultures of Lgr5 + Intestinal Stem Cells and Their Progeny." Nature Methods 11 (1): 106-12. https://doi.org/10.1038/nmeth.2737.

Yui, Shiro, Luca Azzolin, Martti Maimets, Marianne Terndrup Pedersen, Robert P. Fordham, Stine L. Hansen, Hjalte L. Larsen, et al. 2018. "YAP/TAZ-Dependent Reprogramming of Colonic Epithelium Links ECM Remodeling to Tissue Regeneration." Cell Stem Cell 22 (1): 35-49.e7. https://doi.org/10.1016/j.stem.2017.11.001.

Zhao, Hailin, Qian Chen, Azeem Alam, Jiang Cui, Ka Chun Suen, Aurelie Pac Soo, Shiori Eguchi, Jianteng Gu, and Daqing Ma. 2018. "The Role of Osteopontin in the Progression of Solid Organ Tumour." Cell Death and Disease. https://doi.org/10.1038/s41419-018-0391-6. 
1 Smooth muscle-specific MMP17 (MT4-MMP) defines the intestinal ECM niche 2

3 Mara Martín-Alonso ${ }^{1 *}$, Håvard T. Lindholm ${ }^{1, \#}$, Sharif Iqbal $^{2,3, \#}$, Pia Vornewald ${ }^{1 \#}$, Sigrid Hoel ${ }^{1}$,

4 Mirjam J. Damen ${ }^{4}$, A.F.Maarten Altelaar ${ }^{4}$, Pekka Katajisto ${ }^{2,3,5}$, Alicia G. Arroyo ${ }^{6,7}$, Menno J.

5 Oudhoff $^{1 *}$

6

$7 \quad{ }^{1}$ CEMIR - Centre of Molecular Inflammation Research, Department of Clinical and Molecular

8 Medicine, NTNU - Norwegian University of Science and Technology, 7491 Trondheim,

9 Norway

$10{ }^{2}$ Institute of Biotechnology, HiLIFE, University of Helsinki, Finland

$11{ }^{3}$ Molecular and Integrative Bioscience Research Programme, Faculty of Biological and

12 Environmental Sciences, University of Helsinki, Helsinki, Finland

13 'Biomolecular Mass Spectrometry and Proteomics, Utrecht University, Utrecht, The

14 Netherlands

15 Department of Biosciences and Nutrition, Karolinska Institutet, Stockholm, Sweden

$16{ }^{6}$ Centro de Investigaciones Biológicas Margarita Salas (CIB-CSIC), Madrid, Spain

$17{ }^{7}$ Vascular Pathophysiology Area, Centro Nacional de Investigaciones Cardiovasculares

18 (CNIC), Madrid, Spain.CNIC, Madrid, Spain

\#These authors contributed equally to this work

*corresponding authors, e-mail: mara.m.alonso@ntnu.no, menno.oudhoff@ntnu.no

\section{SUPPLEMENTARY FILE}



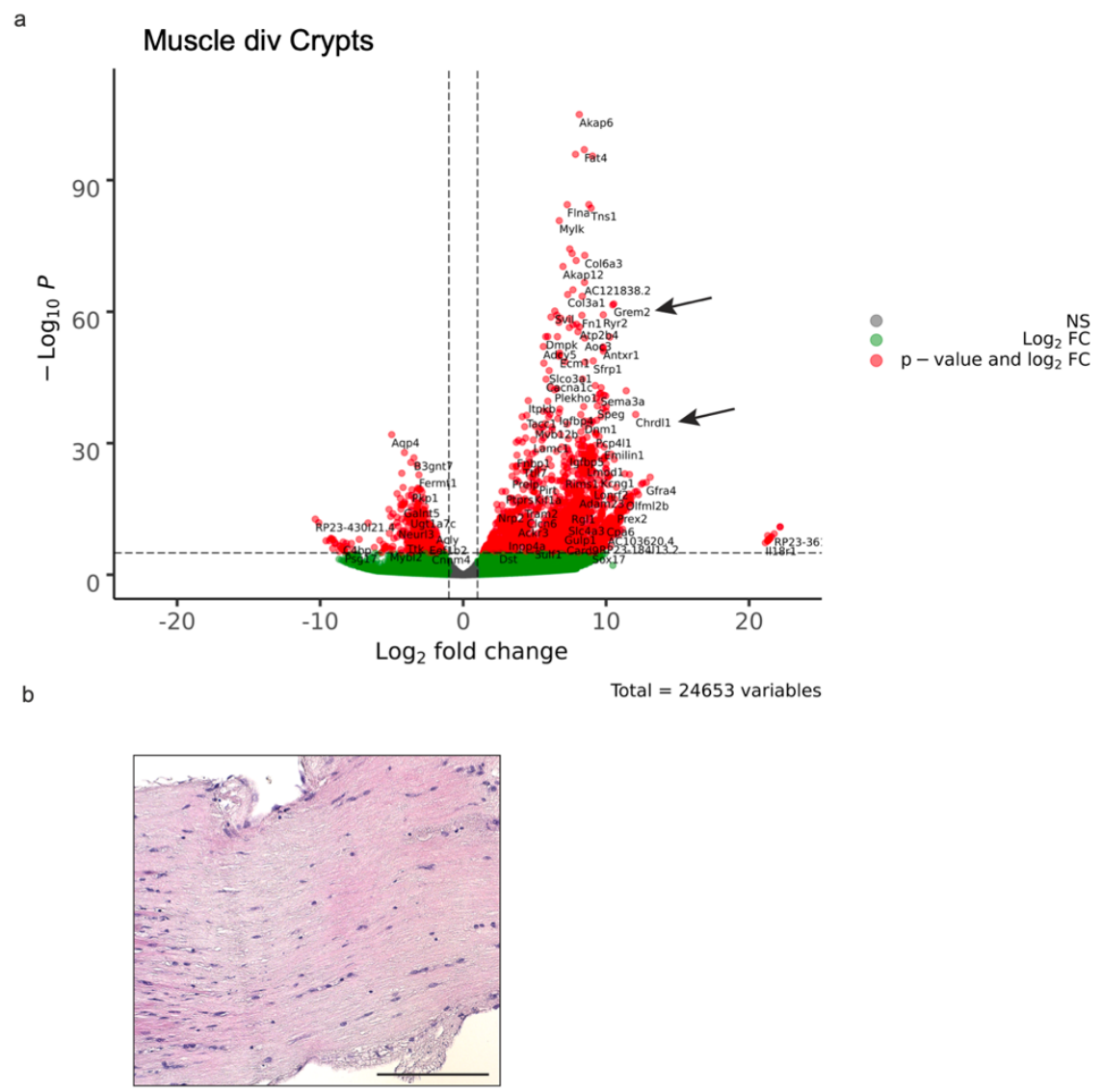

Figure S1. RNAseq of intestinal crypts vs muscle reveals the unique expression of growth

factors by muscle. a Volcano plot showing differential expression of genes between muscle and crypts as Gremlins and Chordin like1 (arrows), BMP signaling antagonists. $\mathrm{n}=3$ biological replicates b. Image of H\&E stained colon muscle strip used to obtain muscle-SN (24h after isolation). Scale 150 $\mu \mathrm{m} . \mathrm{n}=3$.

a

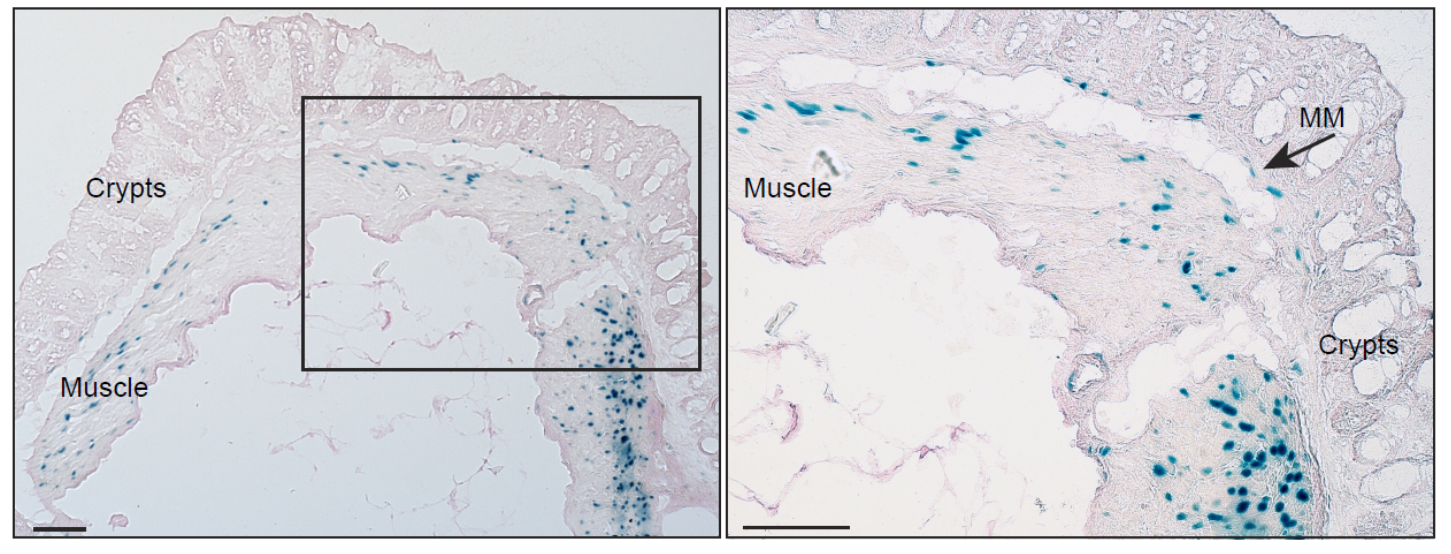

Figure S2. Mmp17 promoter is active in muscle cells from muscularis mucosa and circular and

longitudinal muscle. a. Representative image of a transverse colon cut stained for $\beta$-Galactosidase activity (blue). Scale bar $100 \mu \mathrm{m}$. 


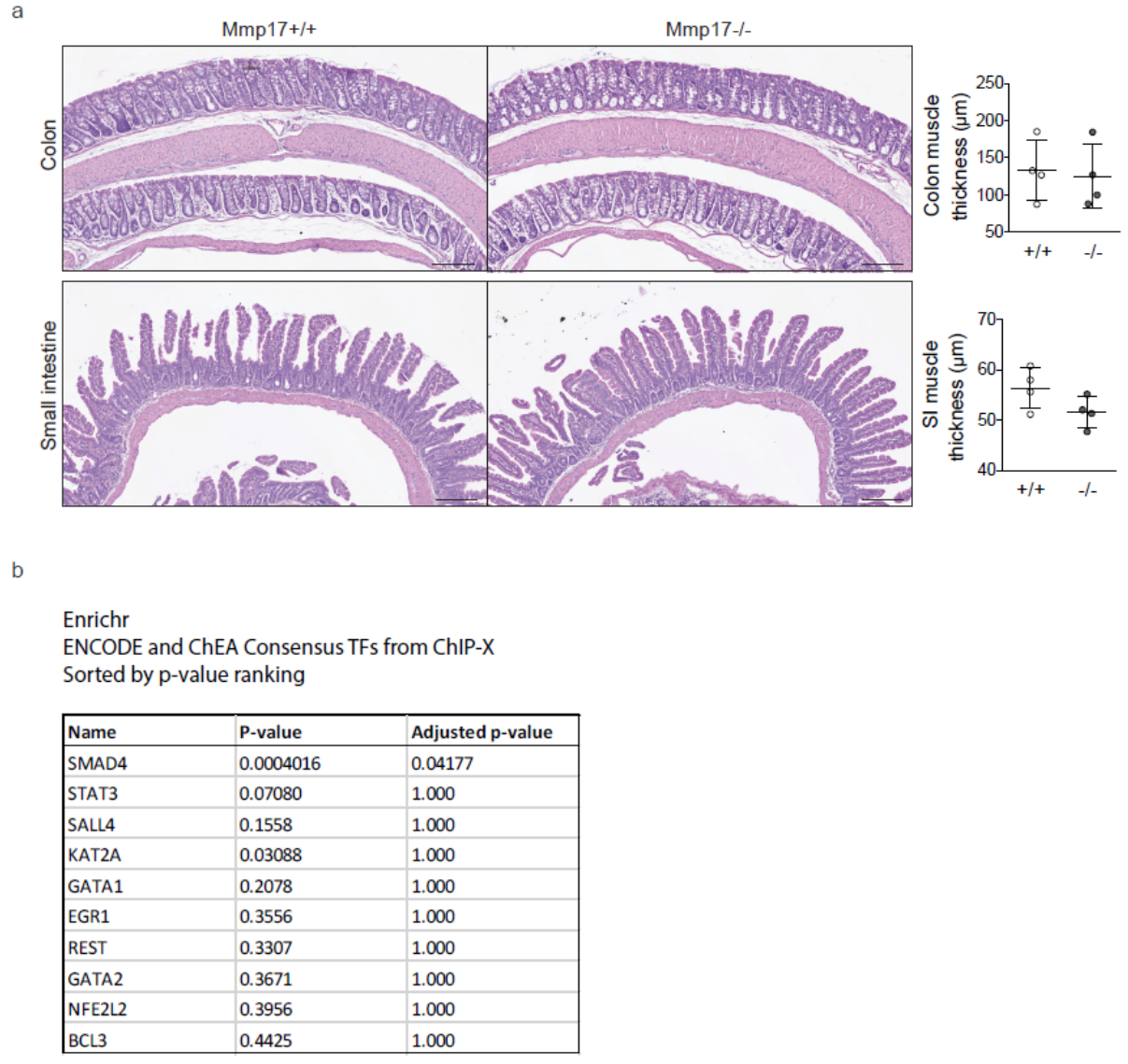

Figure S3. No structural differences in KO muscle and ENRICHR results on transcription factors differences between WT and KO crypts a. Representative H\&E images of transverse colon and small intestine (SI) tissues. Scale $150 \mu \mathrm{m} . \mathrm{n}=4$ mice per genotype. Graphs represent average values for muscle thickness along the swiss roll. b. List of transcription factors altered when comparing WT and KO epithelium (Enrichr). Data in a was analyzed using Mann-Whitney test.

a
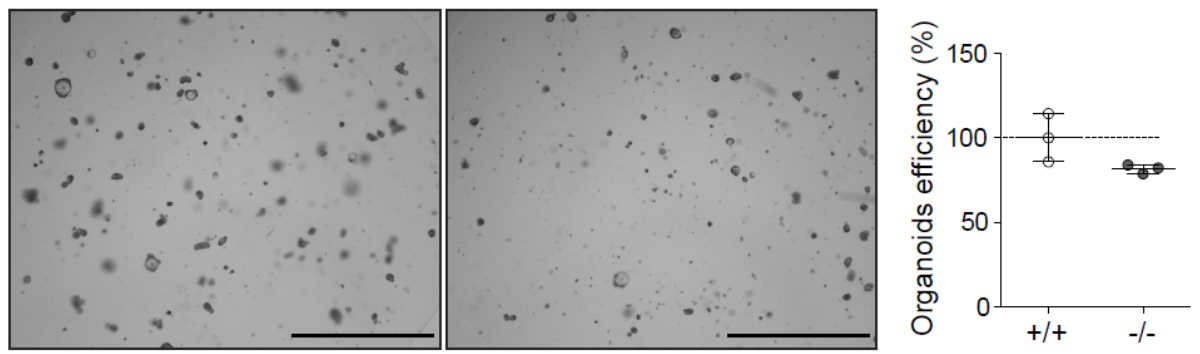

Figure S4. Lower levels in stem cell marker Olfm4 and lower organoids efficiency formation in Mmp17-/- small intestine a. Bright field representative images of small intestine organoids $72 \mathrm{~h}$ after crypt isolation. Graph represents organoids efficiency after $72 \mathrm{~h}$ of WT or KO crypt culture. $n=3$ wells analyzed. Scale $650 \mu \mathrm{m}$. Data in a are means \pm SD and were analyzed using Mann-Whitney test. 

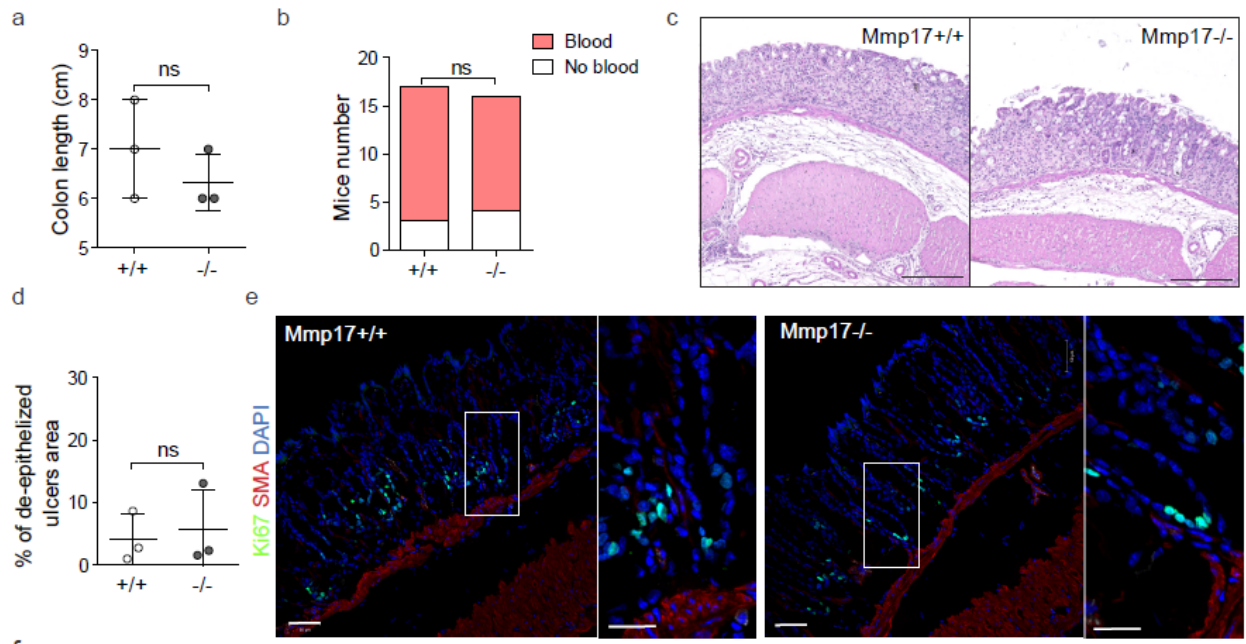

f

g
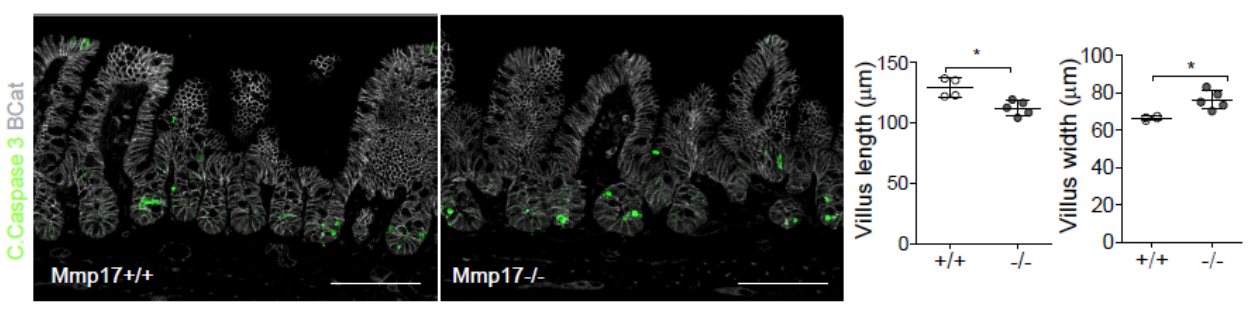

Figure S5. Intestinal damage models produce same injury level in WT and KO mice. a-d.

Graphs represent colon length and presence of blood in stool at D5. $n=3$ mice per genotype in a and $\mathrm{d}$ and 17 and 16 mice in b. c. Representative H\&E picture of a transverse colon cut from mice treated with DSS 3.5\% for 5 days. Scale $200 \mu \mathrm{m}$. n= 3 mice per genotype. $d$. Graph represents the presence of ulcers in the mucosa as a percentaje of the total swiss roll length at $D 5 . n=3$ mice per genotype. e. Representative confocal images stained for Ki67 (green), SMA (red) and nuclei (blue), showing the equivalent reduction in proliferative cells at D5 of DSS treatment in WT and KO. Scale $50 \mu \mathrm{m} ; 25 \mu \mathrm{m}$ in magnified view to the right. $n=3$ mice per genotype. f. Representative confocal image showing Cleaved Caspase 3 staining (green) predominantly at the bottom of the crypts in ileum (24h after irradiation). $\beta$ Cat staining was performed to highlight epithelial cells. Scale bar $100 \mu \mathrm{m} . n=4$ mice per genotype. g. Graph represents villi length and width in small intestinal tissue 3 days after irradiation. $\mathrm{n}=10$ mice per genotype in two independent experiments, analyzed 30 to $40 \mathrm{crypts} / \mathrm{villi}$ per mouse. Numerical data in $a, d$ and $g$ are means \pm SD. Data were analyzed by Mann-Whitney test $(a, d$ and $g)$ and one-tailed Fisher exact test in b. 

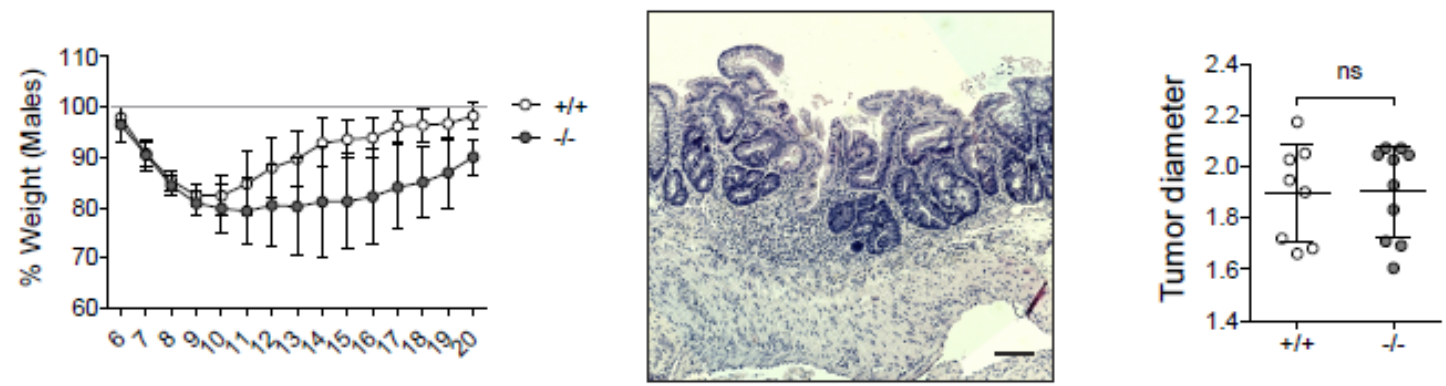

d

ApcMin+

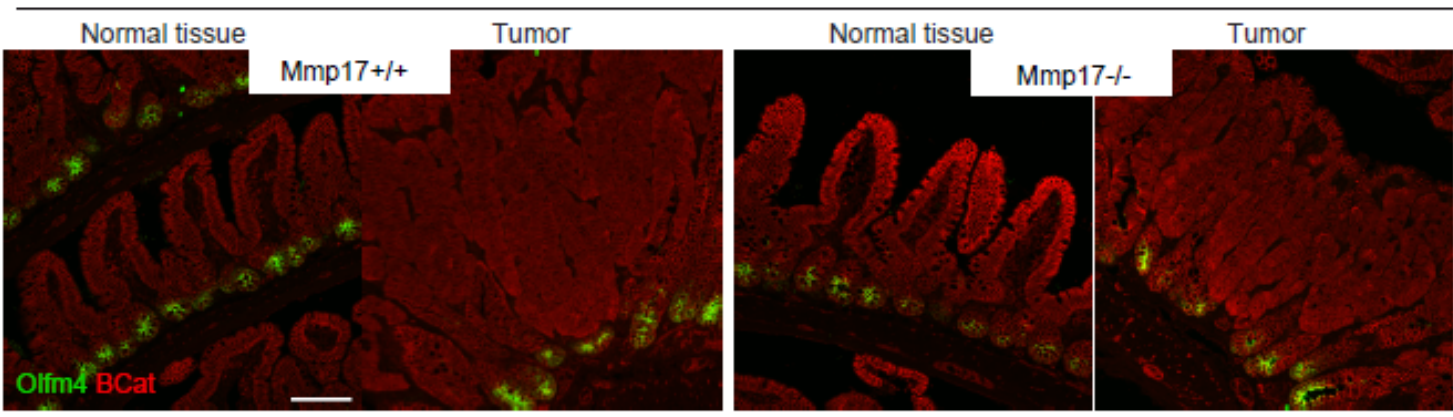

$\mathrm{e}$

ApcMin+Mmp17+/-
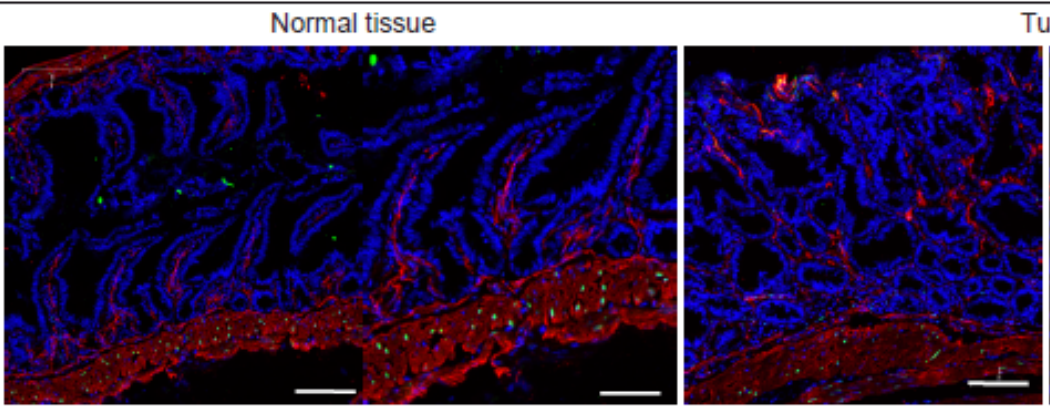

umor

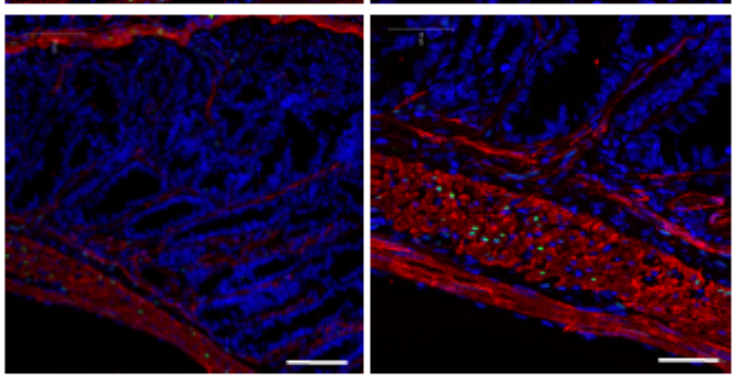

Figure S6. Mmp17 loss in ApcMin background predispose to tumors with no structural differences between WT and KO. a. Graph shows weight evolution in males after 5 days of DSS treatment. $n=4$ mice per genotype. b. Representative H\&E image of crypt reactive atypia in a KO mouse treated with DSS (long-term experiment). Scale bar $100 \mu \mathrm{m}$. $n=6-8$ mice per genotype. c. Graph represents average tumor diameter $(\mathrm{mm})$ in small intestines of ApcMin+ Mmp17 WT or KO. d. Confocal maximum projection images of normal vs tumor area stained for SC marker Olfm4 (Green) and $\beta$ Cat (Red). Scale bar $100 \mu \mathrm{m}$. $n=3-5$ mice per genotype. e. Representative confocal maximum projection images showing $\beta-G a l$ staining (Green) in normal tissue vs tumor areas. B-Gal signal was 
bioRxiv preprint doi: https://doi.org/10.1101/2020.06.18.147769; this version posted June 18, 2020. The copyright holder for this preprint (which was not certified by peer review) is the author/funder. All rights reserved. No reuse allowed without permission.

74 only found in muscle cells (SMA+, Red). Scale bar $100 \mu$ m. $n=3$ mice. Data are means $\pm S D$,

75 numerical data in a was analyzed by two way ANOVA followed by Bonferroni post-test and t-test was

76 applied in c. 


\section{MATERIAL AND METHODS}

\section{Mice}

Mmp17-/- mice in the C57BL/6 background have been described previously (Rikimaru et al. 2007). Mice were handled under pathogen-free conditions in accordance with CoMed NTNU institutional guidelines. Experiments were performed following Norwegian legislation on animal protection and were approved by the local governmental animal care committee. Particularly, experimental designs for DSS, irradiation procedures and ApcMin mice colony handling and tumor evaluation, were approved in advance by Norwegian authorities as stated in FOTS protocols $(11842,15888$ and 17072). Mice included in these protocols were carefully monitored daily or weekly to avoid situations of moderate to high pain and comply with ethical procedures established prior to experiment development in agreement with CoMed facility at NTNU. End point protocols were applied when needed. All mice were genotyped by PCR of earclip samples using the following primers: Mt4-mmp SK1 5'TCAGACACAGCCAGATCAGG-3' SK2 5'- AGCAACACGGCATCCACTAC-3' and SK3 5'AATATGCGAAGTGgACCTGg-3' and ApcMin: 1. 5'-TTCCACTTTGGCATAAGGC-3' 2. 5'GCCATCCCTTCACGTTAG-3' 3. 5'-TTCTGAGAAAGACAGAAGTTA-3'. Experiments were conducted on mice from 8 weeks to 20 weeks of age.

\section{Small intestine and colon crypt isolation}

Small intestinal crypts were isolated and cultured following previously published protocol (Sato and Clevers 2013). Colon organoids were cultured according to a published method (Sato et al. 2011). Briefly, 10 first $\mathrm{cm}$ of the duodenum or the entire colon, were excised, flushed with cold PBS and opened longitudinally. The internal surface of the duodenum was scrapped carefully with a coverslip to remove most of the mucus and part of the intestinal villus. Small pieces of small intestine or colon (2-4 mm in length) were cut with scissors and further washed with ice-cold PBS until the supernatant was clear. Next, tissue fragments were incubated in cold $2 \mathrm{mmol} / \mathrm{L}$ EDTA chelation buffer, for $30 \mathrm{~min}$ (small intestine) to $1 \mathrm{~h}$ (colon) at $4{ }^{\circ} \mathrm{C}$. After removal of the EDTA buffer, tissue fragments were vigorously resuspended in cold PBS (small intestine) or cold chelation buffer (colon) using a 10-mL pipette-BSA coated to isolate intestinal crypts. The tissue fragments were allowed to settle down under normal gravity for 1 minute, and the supernatant was removed for inspection by inverted microscopy. The resuspension/sedimentation procedure was repeated typically 8 times and the supernatants containing crypts (from wash 1 to 8 ) were collected in $50-\mathrm{mL}$ Falcon tubes coated with BSA, through a $70 \mu \mathrm{m}$ cells strainer to remove villi in case of the SI. Isolated crypts were pelleted, washed in PBS, and centrifuged at $200 \mathrm{~g}$ for 3 minutes at 4 ${ }^{\circ} \mathrm{C}$ to separate crypts from single cells. Crypts were resuspended in $10 \mathrm{ml}$ basal crypt medium (BCM, advanced Dulbecco's modified Eagle medium -F12 supplemented with 
penicillin/streptomycin, $10 \mathrm{mM}$ HEPES, $2 \mathrm{mM}$ Glutamax) to quantify its number. After centrifugation and supernatant removal, crypts were resuspended in matrigel (Corning, 7341101 ) and plated in P24 well plates (150 to 250 crypts/well) or 8-well ibidi imaging $\mu$ sildes (80821, Ibidi). Crypts were cultured in ENR (BCM + factors, explained below).

\section{Intestinal organoid culture}

Organoids were cultured in ENR medium consisting of BCM (advanced Dulbecco's modified Eagle medium -F12 supplemented with penicillin/streptomycin, 10 mM HEPES, 2 mM Glutamax) + 1x N2 [ThermoFisher Scientific 100X, 17502048], 1x B-27 [ThermoFisher Scientific 50X, 17504044], and 1x N-acetyl-L-cysteine [Sigma, A7250]) and overlaid with ENR factors containing $50 \mathrm{ng} / \mathrm{ml}$ of murine EGF [Thermo Fisher Scientific, PMG8041], 20\% R-Spondin-CM (conditioned medium, a kind gift from Calvin Kuo, Stanford University School of Medicine, Stanford, CA, USA), $10 \%$ Noggin-CM and, in the case of the colon, with $65 \%$ Wnt-CM (kind gifts from Hans Clevers, Hubrecht Institute, Utrecht, The Netherlands). We also made use of L-WRN (ATCC 3276) cell line conditioned media. Medium was renewed every other day. For passaging, organoids cultures were washed, and matrigel and organoids were disrupted mechanically by strong pipetting, centrifuged at $200 \mathrm{~g}, 5 \mathrm{~min}$ at 4 ${ }^{\circ} \mathrm{C}$ and resuspended in matrigel to plate in P24 wells or 8-well ibidi chambers (80821, Ibidi). In different experiments organoids were exposed to ENR, ER, EN mediums, muscle-SN, obtained as explained below, or human recombinant Periostin (50ng to 500ng) (3548-F2050, R\&D) usually for 4-5 days.

\section{Intestinal muscle isolation and supernatant collection}

Colon tissue was used for muscle isolation, RNA seq, WB or mass spectrometry (MS). After flushing with cold PBS, colons were open in longitudinal and dissected under a bench scope. Using a coverslip, mucosal tissue was completely removed carefully under the dissection scope and muscle was deep frozen in N2 (for RNA seq or WB analysis) or cut in 2-4 cm pieces, wash with sterile PBS twice, spin down and collected in P24 well plates. Pieces of muscle were inspected under the microscope for residual crypts or fat and further cleaned. Pieces were then incubated in DMEM-F12 for $24 \mathrm{~h}$ before collecting the supernatant (muscle$\mathrm{SN})$. Muscle-SN was filtered $(0,20 \mu \mathrm{m})$ and used directly + ENR factors in organoids cultures (right after splitting), sent for MS evaluation or frozen at $-80^{\circ} \mathrm{C}$. Organoids were exposed to muscle-SN for 4-5 days. Muscle pieces were fixed in paraformaldehyde (PFA) $4 \%$ and processed in paraffin for H\&E. Tissues were inspected to confirm the absence of mucosa.

\section{Quantification and imaging of organoids}


P24 well plates were imaged using automatized Z-stack in EVOS2 microscope with CO2, temperature and humidity-controlled incubation chamber (Thermo-Fisher Scientific). Organoid area and classification were evaluated using a custom analysis program written in python based on opencv2 (Lindholm et al., 2020). Images where autoscaled, and a canny edge detection algorithm was run on each individual z-plane using the cv2.canny function. Small pixel groups where removed and a minimal projection of the edges was generated. The contour of objects was defined based on this image. A watershed algorithm was used to split somewhat overlapping objects from each other and the object center was defined as the pixel furthest from the edge of the object. Each object was extracted in a 120x120 image on a white background and classified as either "Junk", "Budding" or "Spheroid" in a neural network implemented and trained using Tensorflow and Keras. A custom visual classification editor was then used to correct locations of organoid centers and the classification group. The order of images was randomized, and treatment information hidden during the classification correction. The images then went through a second segmentation step where the segmentation was re-done as described above. The objects of contours with multiple centers were then split apart with a watershed algorithm that used the corrected organoid centers to split overlapping organoids. Organoids formation efficiency was calculated by manually counting the number of successful organoids 24,48 and $72 \mathrm{~h}$ after crypt isolation in EVOS2 bright field pictures. Organoids size was evaluated from day 1 to 5 . For muscle-SN experiments quantification was performed at day 4 and at day 3 in POSTN treatments.

\section{Immunofluorescence staining in organoids and imaging}

For immunofluorescent labeling and imaging, organoids were grown in 70\% Matrigel-30\% ENR on eight-chamber lbidi $\mu$ slides (80821, Ibidi). Organoids were fixed in PBS containing $4 \%$ paraformaldehyde $(\mathrm{pH} 7.4)$ and $2 \%$ sucrose for $30-45 \mathrm{~min}$, permeabilized, and blocked in PBS-Triton X-100 0.2\%, 2\% normal goat serum (NGS), 1\% BSA Glycine $100 \mu \mathrm{M}$ for $1 \mathrm{~h}$ at RT. Next, organoids were incubated with primary antibodies against the following antigens diluted in PBS-TX100 0.2\% + BSA 0.5\% + NGS 1\%: Ki67 (1:200, rabbit monoclonal antibody (mAb), Invitrogen, MA5-14520), $\beta$-catenin (1:200, mouse mAb, BD Biosciences, 610154), and YAP $\left(1: 100\right.$, rabbit $m A b$, Cell Signalling, 14074) overnight at $4^{\circ} \mathrm{C}$ with slow agitation. Organoids were washed in PBS containing $0.1 \%$ Tween20 and incubated overnight in the same buffer at $4^{\circ} \mathrm{C}$ with the appropriate Alexa Fluor secondary antibody (1:500) along with Hoechst 33342 (1:10,000). Organoids were washed with PBS buffer with $0.1 \%$ Tween 20 and mounted using Fluoromount G (ThermoFisher Scientific, 00-4958-02). Organoids were imaged in a Zeiss Airyscan confocal microscope, using a 10x and 20x objective lens. Images were analyzed using Zen black edition software (Zeiss) and maximal projections are shown. 


\section{RNA seq of organoids and tissue}

Pieces of clean colon muscle, colon crypts (obtained using colon crypt isolation protocol previously described), or pooled organoidswere used for RNA isolation. Tissues were first placed in lysis buffer (RLT, provided in RNeasy® Mini Quiagen Kit, 74104) and disrupted with sterile ceramic beads (Magna Lyser green beads tubes 03358941001) using a Tissue Lyser (FastPrep-24 ${ }^{\mathrm{TM}}$, SKU 116004500), with two rounds of $6500 \mathrm{rpm}$ for 30 seconds each, with care taken to maintain the sample cold. Organoid wells were first disrupted by strong pipetting and pelleted before lysis. RNA isolation was performed following manufacturer instructions (RNeasy® Mini Kit, Qiagen, 74104, for tissue and Direct-zol ${ }^{\mathrm{TM}}$ RNA MiniPrep, BioSite R-2052, for organoids). RNA was quantified by spectrophotometry (ND1000 Spectrophotometer, NanoDrop, Thermo Scientific) and $25 \mu \mathrm{l}$ at a $50 \mathrm{ng} / \mu \mathrm{l}$ concentration of RNA were used for RNA seq. Library preparation and sequencing for tissue RNA seq was performed by NTNU Genomic Core facility. Lexogen SENSE mRNA library preparation kit was used to generate the library and samples were sequenced at $2 \times 75$ bp paired end using Illumina NS500 flow cells. Library preparation and sequencing for organoid RNA seq was performed by Novogene (UK) Co. NEB Next ${ }^{\circledR}$ Ultra ${ }^{\text {TM }}$ RNA Library Prep Kit was used to generate the library and samples where sequenced at 150x2 bp paired end using a Novaseq 6000 (Illumina). The STAR aligner was used to align reads to the Mus musculus genome build mm10(Dobin et al. 2013), (Frankish et al. 2019). featureCounts was used to count the number of reads that uniquely aligned to the exon region of each gene in GENCODE annotation M18 of the mouse genome(Liao, Smyth, and Shi 2014). Genes that had a total count of less than 10 were filtered out. DESeq2 with default settings was used to do a differential expression analysis(Love, Huber, and Anders 2014). Heatmaps were generated using the R-package pheatmap(Kolde 2015). Principal component analysis (PCA) analysis was done with the scikit-learn package using the function sklearn.decomposition.PCA(Pedregosa et al. 2011). Gene set enrichment analysis (GSEA) was performed on the full list of genes from differential expression sorted by log2 fold change and with log2 fold change as weights. GSEA was run with the $\mathrm{R}$ package clusterProfiler using 10000 permutations and otherwise default settings(Yu et al. 2012). A list of the top 250 genes upregulated in crypts was used for Enrichr analysis(Chen et al. 2013; Kuleshov et al. 2016). muscle and crypt RNA seq: E-MTAB-9180 ;ENR vs MuscleSN treated organoids RNA seq: E-MTAB9181. 
Muscle supernatants were collected as stated above. Proteins were reduced with 4 mM DTT at room temperature for 1 hour, and alkylated with $8 \mathrm{mM}$ iodoacetamide at room temperature for $30 \mathrm{~min}$ in the dark, after which additional $4 \mathrm{mM}$ DTT was added. A first digestion was carried out with $40 \mathrm{ng}$ Lys-C at $37^{\circ} \mathrm{C}$ for $4 \mathrm{~h}$. The samples were diluted four times and further digested with $40 \mathrm{ng}$ trypsin at $37^{\circ} \mathrm{C}$ overnight. Digested protein were desalted using Sep-Pak C18 cartridges (Waters), dried by vacuumcentrifuge and stored at $-20{ }^{\circ} \mathrm{C}$ for further use.

Before analysis the peptides were desolved in $2 \%$ formic acid. The digested samples were injected on an Orbitrap Q Exactive HF spectrometer (Thermo Scientific) connected to a UHPLC 1290 system (Agilent). Reconstituted peptides were trapped on a double-fritted (Dr

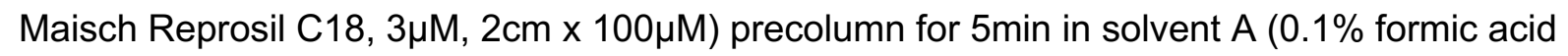
in water) before being separated on an analytical column (Agilent Poroshell, EC-C18, 2.7 $\mu \mathrm{M}$, $50 \mathrm{~cm} \times 75 \mu \mathrm{M})$. Solvent $A$ consisted of $0.1 \%$ formic acid, solvent $B$ of $0.1 \%$ formic acid in $80 \%$ acetonitrile. A 95 min gradient of $13-44 \%$ buffer B followed by $44-100 \%$ B in $3 \mathrm{~min}$, $100 \%$ B for 1 min was applied. MS data were obtained in data-dependent acquisition mode. Full scan MS spectra from m/z $375-1600$ were acquired at a resolution of 60.000 to a target value of $3 \times 106$ or a maximum injection time of $20 \mathrm{~ms}$. The top 15 most intense precursors with a charge state of $2+$ to $5+$ were chosen for fragmentation. HCD fragmentation was performed at 27 normalized collision energy on selected precursors with $16 \mathrm{~s}$ dynamic exclusion at a $1.4 \mathrm{~m} / \mathrm{z}$ isolation window after accumulation to $1 \times 105$ ions or a maximum injection time of $50 \mathrm{~ms}$. Tandem mass spectrometry (MS/MS) spectra were acquired at a resolution of 30.000 .

Proteins IDs and intensity values (abundance) are represented in Table 1.

\section{Tissue immunostaining and immunohistochemistry}

Intestines were open in longitudinal, flushed and rolled into "Swiss rolls" as previously described(Moolenbeek and Ruitenberg 1981). Tissues were then fixed in PFA 4\% 16h and, in the case of frozen samples, included in sucrose $2 \%$ from $3 \mathrm{~h}$ to over night. Swiss rolls were then included in paraffin or in OCT compound for frozen sectioning. Transversal cuts of swiss rolls were used in all experiments. For H\&E staining, paraffin sections of $4 \mu \mathrm{m}$ were subjected to normal deparaffination and hydration. For LacZ staining, frozen sections were stained following $\beta$-gal staining kit indications (K1465-01, Fisher Scientific). Tissue sections were imaged using brightfield microscope EVOS2 with 10 or 20X objective lens and tile scan for the visualization of the complete swiss roll was performed when needed. For immunofluorescence, sections of 4 to $7 \mu \mathrm{m}$ were incubated in blocking buffer for $1 \mathrm{~h}$ (PBS- 
Tx100 0.3\%, NGS 5\%, BSA $2 \%$, Glycine $100 \mu \mathrm{M}$ ), followed by primary antibody incubation overnight. The following antibodies were used for immunofluorescence: anti- $\beta$ Galactosidase ( $\beta$ Gal, Frozen sections, Dil 1:100, Rabbit polyclonal antibody, Abcam, ab4761), anti-Ki67 (Paraffin, 1:200, rabbit monoclonal antibody, Invitrogen, MA5-14520) anti-SMAD4 (Frozen sections, methanol 10min -20C, Dil 1:400, Cell signaling, 46535), anti- $\beta$ catenin (Paraffin, 1:200, Mouse mAb, BD Biosciences, 610154), anti-Olfm4 (Paraffin, 1:200, Rabbit mAb, Cell signaling, 39141), , anti-cleaved caspase 3 (Paraffin, Dil 1:200, Rb pAb, Cell signaling, 9661) and anti-pSMAD1/5/9 (Frozen sections, $10 \mu \mathrm{m}$, Dil 1:800, 13820, Cell Signaling, following TSA amplification, NEL744001KT, Perkin Elmer. following previous specifications(Huycke et al. 2019)). After washing the slides in PBS-Tween $202 \%$, tissues were incubated with the appropriate Alexa Fluor secondary antibody, Anti-SMA-Cy3 directly labeled antibody (1:500, mouse mAb, Sigma-Aldrich C6198) and Hoechst 33342 (1:10,000). Tissue sections were imaged with a Zeiss Airyscan confocal microscope, using a 10x and 20x objective lens. Images were analyzed using Zen black edition software (Zeiss) and maximal projections are shown except for SMAD4 staining (single plane). Tile scans of swiss rolls were performed when required. Percentage of $\beta G$ al positive cells in the muscle was calculated manually in Zen software by counting the total number of nuclei in muscularis mucosa or circular/longitudinal muscle vs $\beta$ Gal positive nuclei in these tissues.

\section{Dextran Sulfate Sodium (DSS) colon injury}

Experimental epithelial colon injury was induced in 8 to 10 weeks mice by supplying $3.5 \%$ dextran sodium sulfate (DSS) (MP Biomedicals, 0216011010) in drinking water ad libitum during 5 days. Before (Day 0 ) and during experimental development (up to day 7 in short protocol or up to 19 days in long-term protocol), mice were monitored daily for signs of stress, pain, body weight loss and presence of blood in stool (detected by hemoFEC, Cobas, 10243744). At day 5, DSS was replaced by regular water to allow epithelial renewal. Control mice to evaluate DSS damage level were euthanized at Day5. For the long-term protocol, weight recovery determined end point (14 days for females and 19 days for males). Colon tissue was then harvested, measured, imaged, fluxed with cold PBS, open in longitudinal and processed for paraffin or OCT, as indicated above. Paraffin sections were stained for H\&E, or Ki67 and SMA. H\&E sections were imaged on EVOS2 (tile scan of complete swiss rolls) and genotype-blind analyzed for signs of injury. Crypt loss, presence of ulcered tissue in the mucosa, signs of immune infiltrate in mucosa and edema were evaluated to create an injury level profile for each sample (Injury Score). The \% of de-epithelialized mucosa was calculated using Fiji (Image $\mathrm{J}$ ) as the total length of intestinal surface devoid of epithelium vs total length of swiss roll. The \% of Ki67+ cells was obtained by quantifying Ki67 positive 
nuclei in mucosa vs total nuclei in mucosa using Cell Profiler. Long-term DSS samples were evaluated by a pathologist to determine presence of reactive atypia.

\section{Irradiation}

8 week-old male mice were subjected to whole body 10Gy irradiation under anesthesia. Weight loss was evaluated at Day 0 and during the length of the experiment ( 3 days). Animals were carefully checked daily for signs of pain or stress. At end point, mice were euthanized and small intestine and colon were harvested, flushed with cold PBS, and processed in swiss rolls for paraffin. H\&E sections were imaged and evaluated blindly for signs of impaired recovery of the mucosal tissue. Parameters as crypt loss, immune infiltrate, crypt length and villus length and width were evaluated to rate the injury score. Villus length and width were measured using Fiji (Image J). We evaluated caspase 3 levels through staining at Day 1 as a positive control for irradiation.

\section{Mmp17-ApcMin tumor evaluation}

The presence and number of intestinal tumors were evaluated in 18 to 20 weeks old ApcMin+Mmp17+/+, Mmp17-/- or Mmp17+/- (females and males) mice. Mice were weekly monitored for weight loss and signs of pain, to avoid situations of moderate to high pain. At end point, mice were euthanized and small intestinal tumors were visualized and counted. Brightfield pictures were taken to double check tumor number and quantify tumor size (Fiji, Image $\mathrm{J}$ ). Tissues were fixed as swiss rolls overnight and processed in paraffin or for OCT as described above 5 um microtome cuts were stained for H\&E and/or for immunostaining. Immunostaining was performed as previously described in paraffin for $\beta$ Catenin and Olfm4 staining. For the evaluation of Mmp17 expression in tumors, Mmp17+/- tissues were processed in OCT and stained for $\beta G a l$ as stated above.

\section{Protein extraction and western blot analysis}

Intestinal tissue (muscle or mucosa tissue isolated as previously described) was disrupted using a tissue lyzer and ceramic beads (Magna Lyser green beads tubes 03358941001) in cold regular RIPA buffer (+ protease and phosphatase inhibitors). Proteins (20 to $100 \mu \mathrm{g}$ ) were resolved by $7-12 \%$ SDS-PAGE in reducing conditions and transferred onto nitrocellulose membranes using iBlot2 ${ }^{\mathrm{TM}}$ dry blotting technology (Thermo Fisher Scientific). Antibodies against SMAD4 (rabbit mAb, Cell signaling, 46535), POSTN (mouse mAb, SAB4200197, MERK, Sigma-Aldrich), $\beta$-tubulin (ab6160, Abcam,) and GAPDH (mouse mAb, Abcam, ab125247) were used at 1:1000 dilution ( $4^{\circ} \mathrm{C}$ overnight). Immunoreactive proteins were visualized with corresponding fluorochrome-conjugated secondary antibodies (680 or 800 ODYSSEY IRDye ${ }^{\circledR}$ ) and recorded by Licor Odyssey technology. Quantification 
of western blot bands was performed using ODYSSEY software (LI-COR Biosciences) and normalized to GAPDH or $\beta$-tubulin levels.

\section{In vitro digestion and western blot}

Human recombinant POSTN (250 ng to $1 \mu \mathrm{g}$; hrPOSTN, 3548-F2-050, R\&D) and the catalytic domain of human recombinant MMP17 (250 ng) (hrMMP17, P4928, Abnova) were incubated in digestion buffer (50 mM Tris- $\mathrm{HCl}, 10 \mathrm{mM} \mathrm{CaCl} 2,80 \mathrm{mM} \mathrm{NaCl},[\mathrm{pH} 7.4])$ for 2 hours at $37^{\circ} \mathrm{C}$. Samples were separated by $12 \%$ SDS-PAGE and transferred to nitrocellulose membranes. Full length hrPOSTN and fragments were detected with an antiPOSTN monoclonal mouse antibody (SAB4200197, MERK, Sigma-Aldrich). An anti-mouse secondary antibody was used to visualize the different fragments.

\section{In situ hybridization (ISH) of intestinal tissues}

ISH using RNAscope technology (ACD, Bio-Techne) was performed using freshly cut paraffin sections of intestinal swiss rolls of $M m p 17+/+$ and $M m p 17-/-$ mice. Chromogenic RNAscope of Lgr5 (Mm-Lgr5, 312171) and Olfm4 (Mm-Olfm4, 311831) in colon and small intestine was performed following manufacturer indications (RNAscope $® 2.5 \mathrm{HD}$ AssayBROWN). Sections were imaged using bright field automatized microscope EVOS2 (tile scan). Quantification of positive Lgr5 cells was performed using Fiji (Image J); total area of positive Lgr5 crypts was normalized to tissue length. Immunofluorescence RNAscope (FISH) was performed following manufacturer protocol (RNAscope $®$ Multiplex Fluorescent v2) and the following probes were used: Mmp17 (Mm-Mmp17-C4 ACD design of NM_011846.5), Grem1 (Mm-Grem1-C3, 314741), Grem2 (473981-C2), Chrdl1 (Mm-Chrdl1, 442811). Zeiss Airyscan scope images are maximal projections of the different channels taken with 10X and 20X objectives.

\section{Statistical analysis}

The statistical analysis performed in each case is explained in detail in the corresponding figure legend, together with the $n$ and the times the experiment was performed. Data were analyzed by two-tailed Student's t-test or by one- or two-way ANOVA followed by Tukey multiple comparison test for data with Gaussian normal distribution. Non-normal distributions were analyzed by Mann-Whitney test. The one-tailed Fisher's exact test was used to analyze the incidence of blood in stool or the presence of reactive atypia. Statistical tests were conducted with Prism 5 software (GraphPad Software, Inc.). Data are presented as mean \pm S.D. and differences were considered statistically significant at $p<0.05$ : ${ }^{*} p$ value $<0.05,{ }^{* *} p$ value $<0.01,{ }^{* * *} p$ value $<0.001$ and ${ }^{* * * *} p$ value $<0.0001$ 
Chen, Edward Y., Christopher M. Tan, Yan Kou, Qiaonan Duan, Zichen Wang, Gabriela V. Meirelles, Neil R. Clark, and Avi Ma'ayan. 2013. "Enrichr: Interactive and Collaborative HTML5 Gene List Enrichment Analysis Tool." BMC Bioinformatics. https://doi.org/10.1186/1471-2105-14-128.

Dobin, Alexander, Carrie A. Davis, Felix Schlesinger, Jorg Drenkow, Chris Zaleski, Sonali Jha, Philippe Batut, Mark Chaisson, and Thomas R. Gingeras. 2013. "STAR: Ultrafast Universal RNA-Seq Aligner." Bioinformatics. https://doi.org/10.1093/bioinformatics/bts635.

Frankish, Adam, Mark Diekhans, Anne Maud Ferreira, Rory Johnson, Irwin Jungreis, Jane Loveland, Jonathan M. Mudge, et al. 2019. "GENCODE Reference Annotation for the Human and Mouse Genomes." Nucleic Acids Research. https://doi.org/10.1093/nar/gky955.

Håvard T. Lindholm, Naveen Parmar, Claire Drurey, Jenny Ostrop, Alberto Díez-Sanchez, Rick M. Maizels, Menno J. Oudhoff. 2020. "Developmental Pathways Regulate Cytokine-Driven Effector and Feedback Responses in the Intestinal Epithelium." BioRxiv.

Huycke, Tyler R., Bess M. Miller, Hasreet K. Gill, Nandan L. Nerurkar, David Sprinzak, L. Mahadevan, and Clifford J. Tabin. 2019. "Genetic and Mechanical Regulation of Intestinal Smooth Muscle Development." Cell. https://doi.org/10.1016/j.cell.2019.08.041.

Kolde, Raivo. 2015. "Pheatmap : Pretty Heatmaps." R Package Version 1.0.8.

Kuleshov, Maxim V., Matthew R. Jones, Andrew D. Rouillard, Nicolas F. Fernandez, Qiaonan Duan, Zichen Wang, Simon Koplev, et al. 2016. "Enrichr: A Comprehensive Gene Set Enrichment Analysis Web Server 2016 Update." Nucleic Acids Research. https://doi.org/10.1093/nar/gkw377.

Liao, Yang, Gordon K. Smyth, and Wei Shi. 2014. "FeatureCounts: An Efficient General Purpose Program for Assigning Sequence Reads to Genomic Features." Bioinformatics. https://doi.org/10.1093/bioinformatics/btt656.

Lindholm, Håvard T., Parmar, Naveen., Drurey, Claire., Ostrop, Jenny., Díez-Sanchez, Alberto., Maizels, Rick M., Oudhoff, Menno J. 2020. "Developmental pathways regulate cytokine-driven effector and feedback responses in the intestinal epithelium" BioRxiv.

Love, Michael I., Wolfgang Huber, and Simon Anders. 2014. "Moderated Estimation of Fold 405 Change and Dispersion for RNA-Seq Data with DESeq2." Genome Biology. https://doi.org/10.1186/s13059-014-0550-8. 
Moolenbeek, C., and E. J. Ruitenberg. 1981. “The 'Swiss Roll': A Simple Technique for Histological Studies of the Rodent Intestine." Laboratory Animals 15 (1): 57-59. https://doi.org/10.1258/002367781780958577.

Pedregosa, Fabian, Vincent Michel, Olivier Grisel, Mathieu Blondel, Peter Prettenhofer, Ron Weiss, Jake Vanderplas, et al. 2011. "Scikit-Learn: Machine Learning in Python Gaël Varoquaux Bertrand Thirion Vincent Dubourg Alexandre Passos PEDREGOSA, VAROQUAUX, GRAMFORT ET AL. Matthieu Perrot." Journal of Machine Learning Research.

Rikimaru, Akiko, Kiyoshi Komori, Takeharu Sakamoto, Hirotake Ichise, Nobuaki Yoshida, Ikuo Yana, and Motoharu Seiki. 2007. "Establishment of an MT4-MMP-Deficient Mouse Strain Representing an Efficient Tracking System for MT4-MMP/MMP-17 Expression in Vivo Using $\beta$-Galactosidase." Genes to Cells. https://doi.org/10.1111/j.13652443.2007.01110.x.

Sato, Toshiro, and Hans Clevers. 2013. "Primary Mouse Small Intestinal Epithelial Cell Cultures." Methods in Molecular Biology. https://doi.org/10.1007/978-1-62703-1257_19.

Sato, Toshiro, Daniel E. Stange, Marc Ferrante, Robert G.J. Vries, Johan H. Van Es, Stieneke Van Den Brink, Winan J. Van Houdt, et al. 2011. "Long-Term Expansion of Epithelial Organoids from Human Colon, Adenoma, Adenocarcinoma, and Barrett's Epithelium." Gastroenterology 141 (5): 1762-72. https://doi.org/10.1053/j.gastro.2011.07.050.

Yu, Guangchuang, Li Gen Wang, Yanyan Han, and Qing Yu He. 2012. "ClusterProfiler: An R Package for Comparing Biological Themes among Gene Clusters." OMICS A Journal of Integrative Biology. https://doi.org/10.1089/omi.2011.0118. 